\title{
Radiation dynamics in fast soliton collisions in the presence of cubic loss
}

\author{
Avner Peleg ${ }^{1}$ and Debananda Chakraborty ${ }^{2}$ \\ 1 Department of Basic Sciences, Afeka College of Engineering, Tel Aviv 69988, Israel and \\ 2 Department of Mathematics, New Jersey City University, Jersey City, NJ 07305, USA
}

(Dated: May 28, 2022)

\begin{abstract}
We study the dynamics of emission of radiation (small-amplitude waves) in fast collisions between two solitons of the nonlinear Schrödinger (NLS) equation in the presence of weak cubic loss. We calculate the radiation dynamics by a perturbation technique with two small parameters: the cubic loss coefficient $\epsilon_{3}$ and the reciprocal of the group velocity difference $1 / \beta$. The agreement between the perturbation theory predictions and the results of numerical simulations with the full coupled-NLS propagation model is very good for large $\beta$ values, and is good for intermediate $\beta$ values. Additional numerical simulations with four simplified NLS models show that the differences between perturbation theory and simulations for intermediate $\beta$ values are due to the effects of Kerr nonlinearity on interpulse interaction in the collision. Thus, our study demonstrates that the perturbation technique that was originally developed to study radiation dynamics in fast soliton collisions in the presence of conservative perturbations can also be employed for soliton collisions in the presence of dissipative perturbations.
\end{abstract}




\section{INTRODUCTION}

The cubic nonlinear Schrödinger (NLS) equation, which describes wave propagation in the presence of cubic (Kerr) nonlinearity and second-order dispersion, is one of the most widely used nonlinear wave models in science. It was successfully employed to describe water wave dynamics [1, 2], nonlinear waves in plasmas [3, 4, Bose-Einstein condensates [5, 6], and propagation of pulses of light through nonlinear optical waveguides [7] 9]. The fundamental NLS solitons are the most important solutions of the cubic NLS equation due to their stability and shape preserving properties. Owing to these properties, fundamental NLS solitons are being considered for applications in many nonlinear optical waveguide systems, including optical waveguide communication links, pulsed waveguide lasers, and optical switches [7, 9-11].

As mentioned above, a major potential application for fundamental NLS solitons is in nonlinear optical waveguide communication links. The rates of transmission of information in optical waveguide links can be significantly enhanced by sending many pulse sequences through the same waveguide [7, 9, 10]. In this multisequence transmission method, the pulses in each sequence propagate with the same central frequency and group velocity, but the central frequency and group velocity are different for pulses from different sequences [7, 9, 10]. Since the pulses in each sequence have the same central frequency, each sequence is called a frequency channel, and multisequence transmission is also called multichannel transmission. Furthermore, since pulses from different sequences propagate with different group velocities, intersequence (interchannel) pulse collisions are very frequent, and can therefore lead to severe transmission degradation. For this reason, many research efforts have been devoted for studying intersequence pulse collisions in general [7, 12, 13], and intersequence soliton collisions in particular [7-10].

Almost all intersequence collisions in multisequence soliton transmission are complete collisions, i.e., collisions in which the two solitons are well-separated before and after the collision [10, 14]. In addition, most intersequence collisions in these systems are fast, that is, the difference between the central frequencies (and group velocities) of the pulses is much larger than the spectral width of each pulse [10, 14, 15]. For this reason, in the current paper, we focus attention on the investigation of complete fast two-soliton collisions. In an ideal optical waveguide, in which the only processes affecting the propagation are due 
to second-order dispersion and Kerr nonlinearity, a complete two-soliton collision is elastic in the sense that the soliton's amplitude, frequency, and shape do not change due to the collision. Furthermore, no radiation is emitted by the colliding solitons. The only effects of a complete fast two-soliton collision in an ideal waveguide are a phase shift proportional to $1 /|\beta|$, and a position shift proportional to $1 /(|\beta| \beta)$, where $\beta$ is the frequency difference between the solitons [14, 16, 17].

However, in real nonlinear optical waveguides, the elastic nature of soliton collisions breaks down due to the presence of additional physical processes, other than second-order dispersion and Kerr nonlinearity. In typical soliton-based multisequence optical waveguide systems, these additional physical processes can be regarded as small perturbations to the cubic NLS equation [7, 9, 10, 16, 17]. In this case, a complete fast two-soliton collision can lead to emission of radiation, changes in the soliton's amplitude, frequency, and shape, and additional phase and position shifts. The magnitude of the collision-induced changes in the soliton parameters and of the amplitude of the emitted radiation is typically proportional to $\epsilon /|\beta|^{n}$, where $\epsilon$ is a small parameter characterizing the strength of the perturbation, and $n$ is a nonnegative integer [16 23]. Collision-induced emission of radiation is of special importance, since it can lead to transmission degradation and to transmission destruction in the following ways. (1) In multisequence transmission, the radiation emitted by solitons from a given sequence resonantly interacts with other sequences [24, 25]. The resonant interaction leads to generation of peaks in the Fourier transforms of the pulse sequences (radiative sidebands) and to corruption and destruction of the soliton patterns [24, 25]. (2) The emitted radiation leads to long-range interaction between solitons from the same sequence [26]. The long-range interaction might cause large relative position shifts between solitons from the same sequence and even undesirable intrasequence soliton collisions [26]. (3) The collision-induced emitted radiation can undergo unstable growth in the presence of Kerr nonlinearity and develop into new (unwanted) solitons [7]. For this reason, it is important to investigate and analyze the dynamics of radiation emitted in complete fast two-soliton collisions.

In previous works, we developed a perturbation technique that describes the effects of weak perturbations on complete fast two-soliton collisions [17, 23, 27]. The perturbation technique is based on the fact that for these collisions, $\epsilon$ and $1 /|\beta|$ are two small parameters. In the first step of the perturbation procedure, we find a soliton solution to the single- 
pulse propagation problem, either in an exact form [23], or in an approximate form, by a perturbation expansion with respect to $\epsilon$ [17, 27, 28]. We then look for a solution of the collision problem in the form of a sum of the two soliton solutions of the single-pulse propagation problems for solitons 1 and 2, plus a term that describes the collision-induced effects. The collisional term is also a sum of two terms $\phi_{1}$ and $\phi_{2}$, which oscillate with the frequencies of solitons 1 and 2, and which describe the collision effects on these solitons. We then substitute the two-pulse solution into the perturbed NLS equation and obtain evolution equations for $\phi_{1}$ and $\phi_{2}$, which we solve by expanding $\phi_{1}$ and $\phi_{2}$ in perturbation series with respect to both $\epsilon$ and $1 /|\beta|$ [17, 23, 27]. The collision-induced changes in the four soliton parameters are calculated by projecting the equations for $\phi_{1}$ and $\phi_{2}$ on the four localized eigenfunctions of the linear operator $\mathcal{L}$, which describes the evolution of small perturbations about the NLS soliton, and by integrating the equations with respect to propagation distance $z$ [17, 23, 27]. Furthermore, the collision-induced radiation dynamics is calculated by projecting the equations for $\phi_{1}$ and $\phi_{2}$ on the nonlocalized eigenfunctions of the linear operator $\mathcal{L}$, and by integrating with respect to $z$ [17, 23, 27].

The perturbation technique of Refs. [17, 23, 27] was originally developed to treat fast twosoliton collisions in the presence of conservative perturbations. Indeed, only in this case, the first step of the perturbation procedure (finding soliton solutions to the single-pulse propagation problem) can be carried out. The perturbation technique was first employed for studying fast two-soliton collisions in the presence of conservative perturbations due to third-order dispersion [17, 27] and quintic nonlinearity [23]. Later on, it was shown that the perturbation approach can also be employed for calculating changes in the four soliton parameters in fast two-soliton collisions in the presence of weak dissipative perturbations, such as nonlinear loss [29, 30] and delayed Raman response [16, 31, 32]. The main idea behind this important extension was that in fast collisions in the presence of dissipative perturbations, the changes in the solitons amplitude and frequency can be approximately described as jumps in the values of these parameters. It follows that the collision-induced changes in soliton parameters can be calculated from the numerical simulations results by subtracting the parameter changes due to single-pulse propagation from the total numerical parameter shifts [29, 30, 32]. This enabled accurate comparison between numerical simulations results and perturbation theory predictions for the changes in soliton parameters up to third order of the perturbation expansion [29, 30, 32]. However, the situation is very 
different for radiation dynamics in fast soliton collisions in the presence of dissipative perturbations. Indeed, since the radiation profile changes both with time and with propagation distance, it is very difficult to accurately subtract radiation-induced changes in the solitons shapes due to single-pulse propagation from the solitons shapes obtained in numerical simulations. The dependence of radiation dynamics on soliton amplitudes, which change during the collision, adds to the difficulty. As a result, it is very difficult to compare the predictions of the perturbation theory and the numerical simulations results for radiation dynamics in fast soliton collisions in the presence of dissipative perturbations. For this reason, despite of its importance, analysis of radiation dynamics in these collisions has not been addressed before.

In the current paper, we address this important problem and provide the first analysis of radiation emission in fast collisions between NLS solitons in the presence of weak dissipative perturbations, considering the cubic loss perturbation as a central example. We assume an optical waveguide setup, in which the effects of cubic loss on single-pulse propagation can be neglected [33]. Therefore, the dynamics of the fast two-soliton collision is described by a perturbed coupled-NLS model, where the perturbation terms are due to the effects of cubic loss on two-pulse interaction. Since the effects of cubic loss on single-pulse propagation are negligible, the soliton solutions of the single-pulse propagation problems are simply the fundamental soliton solutions of the unperturbed NLS equation. Thus, the problem of calculating the soliton solutions of the single-pulse propagation problems in the presence of dissipation is circumvented, and the perturbation technique of Refs. [17, 23, 27] can be used.

We employ this perturbation technique to calculate the collision-induced radiation dynamics and the pulse profile. We then compare the perturbation theory predictions with results of numerical simulations with the full coupled-NLS propagation model for two values of the frequency difference $\beta, \beta=20$ and $\beta=10$, representing large and intermediate frequency difference values, respectively. For large $\beta$ values, we obtain very good agreement between the numerical simulations results and the perturbation theory predictions. For intermediate $\beta$ values, we obtain good agreement between the simulations results and the perturbation theory predictions, but the agreement is not as good as the one obtained for large $\beta$ values. To gain further insight into the reasons for the differences between the perturbation theory and the simulations with the full perturbed coupled-NLS model for in- 
termediate $\beta$ values, we carry out numerical simulations with four simplified NLS models. These additional simulations show that the main reason for the observed differences is due to additional emission of radiation, which is induced by the effects of Kerr nonlinearity on interpulse interaction in the collision. Furthermore, the results of the additional simulations with the simplified NLS models provide the first strong evidence that stochastic perturbed NLS models, which were used in Refs. [16, 34, 35] to describe multisequence soliton transmission in broadband nonlinear optical waveguide systems, correctly capture the collision-induced radiation dynamics in these systems.

We choose to study soliton collisions in the presence of cubic loss, since cubic loss is important in many nonlinear optical waveguide systems, and is therefore a central example for nonlinear dissipative perturbations. The optical waveguide's cubic loss can arise due to two-photon absorption (2PA) or due to gain/loss saturation [36 39]. Propagation of optical pulses in the presence of $2 \mathrm{PA}$ or cubic loss has been studied in many previous works [18, 29, 40 47]. The subject gained renewed interest in recent years due to the importance of 2PA in silicon nanowaveguides, which are expected to play a major role in many applications in optoelectronic devices [37 $39,48,49]$. In the current paper, we assume that the effects of cubic loss on single-pulse propagation are much weaker compared with the effects of cubic loss on interpulse interaction. This situation can be realized, for example, in certain nonlinear semiconductor waveguides, in which 2PA associated with the simultaneous absorption of two photons with the same wavelength (degenerate 2PA) is much weaker than 2PA associated with the simultaneous absorption of two photons with different wavelengths (nondegenerate 2PA) [50 52].

The remainder of the paper is organized in the following manner. In Sec. II], we present the calculation of the collision-induced radiation dynamics by the perturbation theory. We also show that it is possible to obtain the radiation dynamics by analyzing an equivalent single-pulse propagation problem. In Sec. III, we present the results of numerical simulations with the full coupled-NLS model and with four simpler NLS models, and compare these results with the perturbation theory predictions. Our conclusions are summarized in Sec. IV. In Appendix A, we present a summary of the adiabatic perturbation theory for the fundamental NLS soliton. Appendix B is devoted to the derivation of the four simpler NLS propagation models used in Sec. III. 


\section{PERTURBATIVE CALCULATION OF COLLISION-INDUCED RADIATION DYNAMICS}

\section{A. Propagation equation and perturbation approach}

We consider the dynamics of a fast collision between two solitons in a nonlinear optical waveguide with weak cubic loss. We assume that the effects of cubic loss on single-pulse propagation are negligible compared with the effects of cubic loss on interpulse interaction, a situation that can be realized, for example, in certain nonlinear semiconductor waveguides [50 52. In addition, we assume that linear loss is compensated for by linear gain, generated by distributed Raman amplification [53 57]. Under these assumptions, the dynamics of the collision can be described by the following perturbed coupled-NLS model [29, 37]:

$$
\begin{aligned}
& i \partial_{z} \psi_{1}+\partial_{t}^{2} \psi_{1}+2\left|\psi_{1}\right|^{2} \psi_{1}+4\left|\psi_{2}\right|^{2} \psi_{1}=-2 i \epsilon_{3}\left|\psi_{2}\right|^{2} \psi_{1}, \\
& i \partial_{z} \psi_{2}+\partial_{t}^{2} \psi_{2}+2\left|\psi_{2}\right|^{2} \psi_{2}+4\left|\psi_{1}\right|^{2} \psi_{2}=-2 i \epsilon_{3}\left|\psi_{1}\right|^{2} \psi_{2},
\end{aligned}
$$

where $\psi_{1}$ and $\psi_{2}$ are the envelopes of the electric fields of the pulses, $z$ is propagation distance, $t$ is time, and $\epsilon_{3}$ is the cubic loss coefficient, which satisfies $0<\epsilon_{3} \ll 1$ [58]. The terms $\partial_{t}^{2} \psi_{j}$ with $j=1,2$ on the left hand side of Eq. (1) are due to second-order dispersion, while the terms $2\left|\psi_{j}\right|^{2} \psi_{j}$ describe the effects of Kerr nonlinearity on single-pulse propagation. In addition, the terms $4\left|\psi_{2}\right|^{2} \psi_{1}$ and $4\left|\psi_{1}\right|^{2} \psi_{2}$ describe the effects of Kerr nonlinearity on interpulse interaction. The terms $-2 i \epsilon_{3}\left|\psi_{2}\right|^{2} \psi_{1}$ and $-2 i \epsilon_{3}\left|\psi_{1}\right|^{2} \psi_{2}$ describe the effects of cubic loss on interpulse interaction. The terms $-i \epsilon_{3}\left|\psi_{j}\right|^{2} \psi_{j}$ with $j=1,2$ are neglected, since the effects of cubic loss on single-pulse propagation are assumed to be very weak. As a result, the single-pulse propagation problems for the two pulses are described by the unperturbed NLS equations $i \partial_{z} \psi_{j}+\partial_{t}^{2} \psi_{j}+2\left|\psi_{j}\right|^{2} \psi_{j}=0$ for $j=1,2$. The fundamental soliton solutions of these equations are given by:

$$
\psi_{s j}(t, z)=\Psi_{j}\left(x_{j}\right) \exp \left(i \chi_{j}\right)=\eta_{j} \exp \left(i \chi_{j}\right) \operatorname{sech}\left(x_{j}\right),
$$

where $x_{j}=\eta_{j}\left(t-y_{j}+2 \beta_{j} z\right), \chi_{j}=\alpha_{j}-\beta_{j}\left(t-y_{j}\right)+\left(\eta_{j}^{2}-\beta_{j}^{2}\right) z$, and $\eta_{j}, \beta_{j}, y_{j}$, and $\alpha_{j}$ are the amplitude, frequency, position, and phase of the $j$ th soliton.

We consider a complete fast collision between two fundamental solitons of the unperturbed NLS equation in the presence of weak cubic loss. For simplicity and without loss 
of generality, we take the initial soliton frequencies as $\beta_{1}(0)=0$ and $\beta_{2}(0)=\beta$. The complete collision assumption means that the two solitons are well separated at $z=0$ and at the final propagation distance $z=z_{f}$. The fast collision assumption means that the frequency difference between the solitons is much larger than the soliton spectral width, i.e., $\left|\beta_{2}(0)-\beta_{1}(0)\right|=|\beta| \gg 1$. These conditions are satisfied for almost all collisions in long-distance soliton-based multichannel transmission experiments in optical fibers [14, 59].

We study the collision dynamics by employing the perturbation approach that was developed in Refs. [17, 23, 27] for analyzing radiation dynamics in fast two-soliton collisions in the presence of conservative perturbations. Following this perturbation approach, we look for a solution of Eq. (1) in the form

$$
\psi_{j}(t, z)=\psi_{j 0}(t, z)+\phi_{j}(t, z)
$$

where $j=1,2, \psi_{j 0}$ are the solutions of Eq. (1) without the interpulse interaction terms, and $\phi_{j}$ represent small corrections to $\psi_{j 0}$ due to the collision. In the absence of the interpulse interaction terms, Eq. (1) reduces to a pair of uncoupled unperturbed NLS equations. Therefore, in the current problem, the $\psi_{j 0}$ are simply the fundamental soliton solutions of the unperturbed NLS equation, i.e., $\psi_{j 0}(t, z)=\psi_{s j}(t, z)$ for $j=1,2$. Note that finding the solutions of the single-pulse propagation problems in the presence of cubic loss becomes unnecessary because of the omission of the terms $-i \epsilon_{3}\left|\psi_{j}\right|^{2} \psi_{j}$ from the propagation equation (due to the assumed weakness of cubic loss effects on single-pulse propagation). In this manner, the application of the perturbation approach of Refs. [17, 23, 27] to soliton collisions in the presence of nonlinear dissipation is enabled, despite of the fact that the approach was originally developed for treating fast soliton collisions in the presence of conservative perturbations. We substitute the ansatz (3) together with the relations $\psi_{j 0}(t, z)=\psi_{s j}(t, z)=$ $\Psi_{j}\left(x_{j}\right) \exp \left(i \chi_{j}\right)$ and $\phi_{j}(t, z)=\Phi_{j}(t, z) \exp \left(i \chi_{j}\right)$ into Eq. (1) and obtain equations for the $\Phi_{j}[17,23,29]$. We focus attention on the calculation of $\Phi_{1}$, since the calculation of $\Phi_{2}$ is similar. The equation for $\Phi_{1}$ is

$$
\begin{aligned}
& i \partial_{z} \Phi_{1}+\left[\left(\partial_{t}^{2}-\eta_{1}^{2}\right) \Phi_{1}+4\left|\Psi_{1}\right|^{2} \Phi_{1}+2 \Psi_{1}^{2} \Phi_{1}^{*}\right] \\
& =-4\left[\left|\Psi_{2}\right|^{2} \Psi_{1}+\left|\Psi_{2}\right|^{2} \Phi_{1}+\Psi_{1}\left(\Psi_{2} \Phi_{2}^{*}+\Psi_{2}^{*} \Phi_{2}\right)\right] \\
& -2 i \epsilon_{3}\left[\left|\Psi_{2}\right|^{2} \Psi_{1}+\left|\Psi_{2}\right|^{2} \Phi_{1}+\Psi_{1}\left(\Psi_{2} \Phi_{2}^{*}+\Psi_{2}^{*} \Phi_{2}\right)\right] .
\end{aligned}
$$

We solve Eq. (4) and the corresponding equation for $\Phi_{2}$ by expanding the $\Phi_{j}$ in per- 
turbation series with respect to $\epsilon_{3}$ and $1 / \beta$. More specifically, the expansions of the $\Phi_{j}$ are

$$
\begin{aligned}
& \Phi_{j}(t, z)=\Phi_{j 1}^{(0)}(t, z)+\Phi_{j 1}^{(1)}(t, z)+\Phi_{j 2}^{(0)}(t, z) \\
& +\Phi_{j 2}^{(1)}(t, z)+\ldots,
\end{aligned}
$$

where the first subscript in $\Phi_{j k}^{(m)}$ stands for the pulse index, the second subscript indicates the combined order with respect to both $\epsilon_{3}$ and $1 / \beta$, and the superscript represents the order in $\epsilon_{3}$. Substitution of the expansions (5) into Eq. (4) yields linear equations for the $\Phi_{1 k}^{(m)}$, which can be integrated with respect to $z$. The collision-induced changes in the four soliton parameters are calculated by projecting both sides of the resulting equations on the four localized eigenfunctions of the linear operator $\mathcal{L}$, which describes the evolution of small perturbations about the fundamental soliton of the unperturbed NLS equation (see Appendix A and Refs. [17, 23, 26, 60, 61] for a description of the operator $\mathcal{L}$ and its eigenfunctions). The dynamics of the collision-induced radiation emitted by soliton 1 is calculated by projecting the equations for the $\Phi_{1 k}^{(m)}$ on the nonlocalized eigenfunctions of $\mathcal{L}$. This calculation is described in sections 【B and 【C.

The only effect of the collision on soliton 1 in order $1 / \beta$ is a phase shift, which is given by [14, 16, 17]:

$$
\Delta \alpha_{11}^{(0)}=4 \eta_{2} /|\beta|
$$

In addition, the only collision-induced effect on soliton 1 in order $1 / \beta^{2}$ is a position shift, which is given by [14, 16, 17]:

$$
\Delta y_{12}^{(0)}=4 \eta_{2} /(\beta|\beta|)
$$

Both effects are caused by the term $4\left|\psi_{2}\right|^{2} \psi_{1}$ in Eq. (1) [14, 17]. That is, the collisioninduced effects in orders $1 / \beta$ and $1 / \beta^{2}$ are due to Kerr-induced interpulse interaction and not due to cubic loss. In addition, there are no terms of order $\epsilon_{3} \beta$ on the right hand side of Eq. (4) and therefore, there are no collision-induced effects in order $\epsilon_{3}$. We therefore start the detailed description of the perturbative calculations by considering the effects of the collision in order $\epsilon_{3} / \beta$. 


\section{B. Basic calculation of radiation dynamics in order $\epsilon_{3} / \beta$}

In order $\epsilon_{3} / \beta$, Eq. (4) reduces to

$$
\partial_{z} \Phi_{12}^{(1)}=-2 \epsilon_{3}\left|\Psi_{2}\right|^{2} \Psi_{1}=-\frac{2 \epsilon_{3} \eta_{1} \eta_{2}^{2}}{\cosh \left(x_{1}\right) \cosh ^{2}\left(x_{2}\right)} .
$$

We denote by $\Delta z_{c}$ and $z_{c}$ the collision interval and the collision distance. The collision interval $\Delta z_{c}$ is the interval along which the envelopes of the colliding solitons overlap. It can be estimated by $\Delta z_{c}=1 /(2|\beta|)$. The collision distance $z_{c}$ is the distance at which the maxima of $\left|\psi_{j}(t, z)\right|$ coincide. This distance is given by $z_{c}=\left[y_{2}(0)-y_{1}(0)\right] /(2 \beta)$, where $y_{1}(0)$ and $y_{2}(0)$ are the initial positions of the solitons. In a fast collision, the collision takes place in a small interval $\left[z_{c}-\Delta z_{c} / 2, z_{c}+\Delta z_{c} / 2\right]$ around $z_{c}$. Thus, to calculate the collisioninduced effects in order $\epsilon_{3} / \beta$, we integrate both sides of Eq. (8) over the collision region. This integration yields:

$$
\Phi_{12}^{(1)}\left(t, z_{c}+\Delta z_{c} / 2\right)=-\frac{2 \epsilon_{3} \eta_{1} \eta_{2}^{2}}{\cosh \left(x_{1}\right)} \int_{z_{c}-\Delta z_{c} / 2}^{z_{c}+\Delta z_{c} / 2} \frac{d z^{\prime}}{\cosh ^{2}\left(x_{2}\right)},
$$

where we used $\Phi_{12}^{(1)}\left(t, z_{c}-\Delta z_{c} / 2\right) \simeq 0$. Since the integrand on the right hand side of Eq. (9) is sharply peaked at a small interval around $z_{c}$, we can extend the integral's limits to $-\infty$ and $\infty$. The integration yields the following expression for $\Phi_{12}^{(1)}\left(t, z_{c}+\Delta z_{c} / 2\right)$ :

$$
\Phi_{12}^{(1)}\left(t, z_{c}+\Delta z_{c} / 2\right)=-\frac{2 \epsilon_{3} \eta_{1} \eta_{2}}{|\beta| \cosh \left(x_{1}\right)} .
$$

We now write $\Phi_{12}^{(1)}\left(t, z_{c}+\Delta z_{c} / 2\right)$ and its complex conjugate in a vector form and expand this vector in terms of the eigenfunctions of the linear operator $\mathcal{L}$ :

$$
\begin{aligned}
& \left(\begin{array}{c}
\Phi_{12}^{(1)}\left(t, z_{c}+\Delta z_{c} / 2\right) \\
\Phi_{12}^{(1) *}\left(t, z_{c}+\Delta z_{c} / 2\right)
\end{array}\right)=-\frac{2 \epsilon_{3} \eta_{1} \eta_{2}}{|\beta| \cosh \left(x_{1}\right)}\left(\begin{array}{l}
1 \\
1
\end{array}\right) \\
& =\sum_{j=0}^{3} \tilde{a}_{j} f_{j}\left(x_{1}\right)+\left(\begin{array}{c}
v_{12 b}\left(t, z_{c}+\Delta z_{c} / 2\right) \\
v_{12 b}^{*}\left(t, z_{c}+\Delta z_{c} / 2\right)
\end{array}\right) .
\end{aligned}
$$

In Eq. (11), $\tilde{a}_{j}$ with $j=0, \ldots, 3$ are constants, $f_{j}\left(x_{1}\right)$ with $j=0, \ldots, 3$ are the four localized eigenfunctions of $\mathcal{L}$, defined by Eq. (A5), and $v_{12 b}(t, z)$ is the radiation emitted by soliton 1 in this basic version of the perturbative calculation. The radiation part $v_{12 b}(t, z)$ is expressed by the following expansion in the nonlocalized eigenfunctions $f_{s}\left(x_{1}\right)$ and $\bar{f}_{s}\left(x_{1}\right)$ 
of the operator $\mathcal{L}$ :

$$
\begin{aligned}
& \left(\begin{array}{l}
v_{12 b}\left(t, z_{c}+\Delta z_{c} / 2\right) \\
v_{12 b}^{*}\left(t, z_{c}+\Delta z_{c} / 2\right)
\end{array}\right)= \\
& \int_{-\infty}^{+\infty} \frac{d s}{2 \pi}\left[a_{s}\left(z_{c}+\Delta z_{c} / 2\right) f_{s}\left(x_{1}\right)+a_{s}^{*}\left(z_{c}+\Delta z_{c} / 2\right) \bar{f}_{s}\left(x_{1}\right)\right]
\end{aligned}
$$

where $-\infty<s<\infty$, and the eigenfunctions are defined in Eqs. A8 and A9. The collision-induced changes in the four soliton parameters are calculated by projecting both sides of Eq. (11) on the four localized eigenfunctions of $\mathcal{L}$. This calculation shows that the only effect of the collision on the soliton parameters in order $\epsilon_{3} / \beta$ is an amplitude shift, $\Delta \eta_{12}^{(1)}$, which is given by [29]:

$$
\Delta \eta_{12}^{(1)}=-4 \epsilon_{3} \eta_{1} \eta_{2} /|\beta|
$$

Furthermore, the radiation part of $\Phi_{12}^{(1)}$ at $z=z_{c}+\Delta z_{c} / 2, v_{12 b}\left(t, z_{c}+\Delta z_{c} / 2\right)$, is obtained by projecting both sides of Eq. (11) on the nonlocalized eigenfunctions of $\mathcal{L}$. This calculation yields the following expression for $a_{s}\left(z_{c}+\Delta z_{c} / 2\right)$ :

$$
a_{s}\left(z_{c}+\Delta z_{c} / 2\right)=\frac{2 \pi \epsilon_{3} \eta_{1} \eta_{2}(s+i)^{2}}{|\beta|\left(s^{2}+1\right) \cosh (\pi s / 2)} .
$$

Outside of the collision interval, that is, for distances $z>z_{c}+\Delta z_{c} / 2$, the two solitons are no longer overlapping. As a result, in this post-collision interval, the term $-2 i \epsilon_{3}\left|\Psi_{2}\right|^{2} \Psi_{1}$ and all other interpulse interaction terms on the right hand side of Eq. (4) are exponentially small and can be neglected. Thus, for $z>z_{c}+\Delta z_{c} / 2$, the equation describing the evolution of $\Phi_{12}^{(1)}$ is:

$$
\partial_{z} \Phi_{12}^{(1)}-i\left[\left(\partial_{t}^{2}-\eta_{1}^{2}\right) \Phi_{12}^{(1)}+4\left|\Psi_{1}\right|^{2} \Phi_{12}^{(1)}+2 \Psi_{1}^{2} \Phi_{12}^{(1) *}\right]=0
$$

This equation and its complex conjugate can be written as

$$
\partial_{z}\left(\begin{array}{c}
\Phi_{12}^{(1)} \\
\Phi_{12}^{(1)}
\end{array}\right)-i \eta_{1}^{2} \mathcal{L}\left(\begin{array}{c}
\Phi_{12}^{(1)} \\
\Phi_{12}^{(1)}
\end{array}\right)=0
$$

Since $\Phi_{12}^{(1)}(t, z)=\sum_{j=0}^{3} \tilde{a}_{j} f_{j 1}\left(x_{1}\right)+v_{12 b}(t, z)$, the equation describing radiation dynamics in the post-collision interval is

$$
\partial_{z}\left(\begin{array}{c}
v_{12 b} \\
v_{12 b}^{*}
\end{array}\right)-i \eta_{1}^{2} \mathcal{L}\left(\begin{array}{c}
v_{12 b} \\
v_{12 b}^{*}
\end{array}\right)=0
$$


We solve Eq. (17) with an initial condition at $z=z_{c}+\Delta z_{c} / 2$, which is given by Eqs. (12) and (14). For this purpose, we first expand $v_{12 b}(t, z)$ in the nonlocalized eigenfunctions of $\mathcal{L}$

$$
\left(\begin{array}{l}
v_{12 b}(t, z) \\
v_{12 b}^{*}(t, z)
\end{array}\right)=\int_{-\infty}^{+\infty} \frac{d s}{2 \pi}\left[a_{s}(z) f_{s}\left(x_{1}\right)+a_{s}^{*}(z) \bar{f}_{s}\left(x_{1}\right)\right] .
$$

Projecting both sides of Eq. (17) on the nonlocalized eigenfunctions of $\mathcal{L}$, while using the expansion (18), we obtain the following equation for the expansion coefficients

$$
\frac{d a_{s}(z)}{d z}-i \eta_{1}^{2}\left(s^{2}+1\right) a_{s}(z)=0
$$

Integrating Eq. (19) with respect to $z$ and using the initial condition (14), we arrive at

$$
a_{s}\left(z \geq z_{c}+\Delta z_{c} / 2\right)=\frac{2 \pi \epsilon_{3} \eta_{1} \eta_{2}(s+i)^{2}}{|\beta|\left(s^{2}+1\right) \cosh (\pi s / 2)} \exp \left[i \eta_{1}^{2}\left(s^{2}+1\right)\left(z-z_{c}\right)\right] .
$$

Thus, the dynamics of the collision-induced radiation emitted by soliton 1 in the postcollision interval is fully described by Eqs. (18) and 200.

The envelope of the electric field of soliton 1 in the post-collision interval is given by: $\psi_{1 b}(t, z)=\psi_{10}(t, z)+\phi_{1 b}(t, z)$, where $\phi_{1 b}(t, z)$ describes the effects of the collision on soliton 1 in the basic version of the perturbation theory. $\psi_{1 b}(t, z)$ can be written as:

$$
\begin{aligned}
& \psi_{1 b}(t, z)=\left\{\frac{\eta_{1}}{\cosh \left(x_{1}\right)}-\Delta \eta_{12}^{(1)} \frac{\left[x_{1} \tanh \left(x_{1}\right)-1\right]}{\cosh \left(x_{1}\right)}+v_{12 b}(t, z)\right\} \\
& \times \exp \left\{i\left[\chi_{1}(t, z)+\Delta \alpha_{11}^{(0)}\right]\right\},
\end{aligned}
$$

where $x_{1}=\eta_{1}\left[t-y_{1}(z)\right], \chi_{1}(t, z)=\alpha_{1}(0)+\eta_{1}^{2} z, \Delta \alpha_{11}^{(0)}$ is given by Eq. (6), $\Delta \eta_{12}^{(1)}$ is given by Eq. 13 , and $\alpha_{1}(0)$ is the initial phase of soliton 1 . In addition, $y_{1}(z)=y_{1}(0)+\Delta y_{12}^{(0)}+y_{1}^{(C)}(z)$, where $\Delta y_{12}^{(0)}$ is given by Eq. (7), and $y_{1}^{(C)}(z)=\left[40 \epsilon_{3} \eta_{1}^{2} \eta_{2}\left(z-z_{c}\right)\right] /(3|\beta| \beta)$ is the position shift arising from the collision-induced frequency shift [see Eq. (B1) in Appendix B].

\section{Taking into account propagation of radiation in the collision interval}

The perturbative calculation described in Sec. IIB is based on neglecting the effects of propagation of radiation in the collision interval. More accurately, in writing Eq. (8), we neglected the terms $-i\left(\partial_{t}^{2}-\eta_{1}^{2}\right) \Phi_{12}^{(1)},-4 i\left|\Psi_{1}\right|^{2} \Phi_{12}^{(1)}$, and $-2 i \Psi_{1}^{2} \Phi_{12}^{(1) *}$, which describe these effects, since these terms are of order $\epsilon_{3} / \beta^{2}$. However, for $|t|>z$, the radiation profile can receive significant contributions from fast moving waves with group velocities larger than $|\beta|$. Therefore, it might be important to include the contributions of these fast moving waves to 
the radiation profile by taking into account propagation of radiation in the collision interval (see also Ref. [23], where a similar calculation was carried out for fast collisions between NLS solitons in the presence of quintic nonlinearity). We therefore turn to describe an improved perturbation approach that achieves this goal.

Following the calculation in Ref. [23], we denote by $\Phi_{12 c}^{(1)}$ the part of $\Phi_{1}$ that describes the collision-induced effects in order $\epsilon_{3} / \beta$ and the effects of propagation of radiation in the collision interval. The equation for the evolution of $\Phi_{12 c}^{(1)}$ is:

$$
\partial_{z} \Phi_{12 c}^{(1)}-i\left[\left(\partial_{t}^{2}-\eta_{1}^{2}\right) \Phi_{12 c}^{(1)}+4\left|\Psi_{1}\right|^{2} \Phi_{12 c}^{(1)}+2 \Psi_{1}^{2} \Phi_{12 c}^{(1) *}\right]=-2 \epsilon_{3}\left|\Psi_{2}\right|^{2} \Psi_{1}
$$

This equation and its complex conjugate can be written as

$$
\partial_{z}\left(\begin{array}{c}
\Phi_{12 c}^{(1)} \\
\Phi_{12 c}^{(1) *}
\end{array}\right)-i \eta_{1}^{2} \mathcal{L}\left(\begin{array}{c}
\Phi_{12 c}^{(1)} \\
\Phi_{12 c}^{(1) *}
\end{array}\right)=-\frac{2 \epsilon_{3} \eta_{1} \eta_{2}^{2}}{\cosh \left(x_{1}\right) \cosh ^{2}\left(x_{2}\right)}\left(\begin{array}{l}
1 \\
1
\end{array}\right)
$$

We express $\Phi_{12 c}^{(1)}$ in the form

$$
\left(\begin{array}{c}
\Phi_{12 c}^{(1)}(t, z) \\
\Phi_{12 c}^{(1) *}(t, z)
\end{array}\right)=\sum_{j=0}^{3} \tilde{a}_{j}^{(c)} f_{j}\left(x_{1}\right)+\left(\begin{array}{l}
v_{12 c}(t, z) \\
v_{12 c}^{*}(t, z)
\end{array}\right)
$$

where the radiation part $v_{12 c}(t, z)$ is expanded in the nonlocalized eigenfunctions of $\mathcal{L}$

$$
\left(\begin{array}{l}
v_{12 c}(t, z) \\
v_{12 c}^{*}(t, z)
\end{array}\right)=\int_{-\infty}^{+\infty} \frac{d s}{2 \pi}\left[a_{s}^{(c)}(z) f_{s}\left(x_{1}\right)+a_{s}^{(c) *}(z) \bar{f}_{s}\left(x_{1}\right)\right]
$$

The expansion of the right hand side of Eq. (23) in the eigenfunctions of $\mathcal{L}$ is

$$
\begin{aligned}
& -\frac{2 \epsilon_{3} \eta_{1} \eta_{2}^{2}}{\cosh \left(x_{1}\right) \cosh ^{2}\left(x_{2}\right)}\left(\begin{array}{l}
1 \\
1
\end{array}\right)=\sum_{j=0}^{3} \tilde{b}_{j} f_{j}\left(x_{1}\right) \\
& +\int_{-\infty}^{+\infty} \frac{\mathrm{d} s}{2 \pi}\left[b_{s}(z) f_{s}\left(x_{1}\right)+b_{s}^{*}(z) \bar{f}_{s}\left(x_{1}\right)\right]
\end{aligned}
$$

where

$$
b_{s}(z)=2 \epsilon_{3} \eta_{1} \eta_{2}^{2} \int_{-\infty}^{\infty} \frac{d x_{1} f_{s}^{+}\left(x_{1}\right) \sigma_{3}}{\cosh \left(x_{1}\right) \cosh ^{2}\left(x_{2}\right)}\left(\begin{array}{l}
1 \\
1
\end{array}\right) .
$$

We substitute the expansions (24)-(27) into Eq. (23) and project both sides of the resultant equation on the nonlocalized eigenfunctions of $\mathcal{L}$. This calculation yields the following equation for the $a_{s}^{(c)}$ coefficients:

$$
\frac{d a_{s}^{(c)}(z)}{d z}-i \eta_{1}^{2}\left(s^{2}+1\right) a_{s}^{(c)}(z)=b_{s}(z)
$$


The solution of Eq. 28) on the interval $\left[z_{c}-\Delta z_{c} / 2, z\right]$ is

$$
a_{s}^{(c)}(z)=\int_{z_{c}-\Delta z_{c} / 2}^{z} d z^{\prime} b_{s}\left(z^{\prime}\right) \exp \left[i \eta_{1}^{2}\left(s^{2}+1\right)\left(z-z^{\prime}\right)\right],
$$

where $a_{s}^{(c)}\left(z_{c}-\Delta z_{c} / 2\right) \simeq 0$ is used. The integrand on the right hand side of Eq. 29 is sharply peaked at a small interval around $z_{c}$. Therefore, we can extend the integral's limits to $-\infty$ and $\infty$ and obtain

$$
a_{s}^{(c)}(z)=\int_{-\infty}^{\infty} d z^{\prime} b_{s}\left(z^{\prime}\right) \exp \left[i \eta_{1}^{2}\left(s^{2}+1\right)\left(z-z^{\prime}\right)\right] .
$$

Substituting Eq. (27) into Eq. (30) and carrying out the integrations we arrive at:

$$
\begin{aligned}
& a_{s}^{(c)}(z)=\frac{-2 \pi^{2} \epsilon_{3} \eta_{1} \eta_{2}}{4|\beta| A B} \frac{(s+i)^{2}(s-B)}{\sinh \left[\frac{\pi\left(s^{2}+1\right)}{4 A}\right] \cosh \left[\frac{\pi\left(s^{2}+1-2 B s\right)}{4 B}\right]} \\
& \times \exp \left[i \eta_{1}^{2}\left(s^{2}+1\right)\left(z-z_{c}\right)\right],
\end{aligned}
$$

where $A=\eta_{2} \beta / \eta_{1}^{2}$ and $B=\beta / \eta_{1}$. It is straightforward to show that in the limit $|\beta| \gg 1$, Eq. (31) reduces to Eq. (20). Equations (25) and (31) describe the evolution of the radiation profile $\left|v_{12 c}(t, z)\right|$ in the improved perturbation approach. The envelope of the electric field of soliton 1 in the improved perturbation approach is $\psi_{1 c}(t, z)=\psi_{10}(t, z)+\phi_{1 c}(t, z)$, where $\phi_{1 c}(t, z)$ describes the effects of the collision in this version of the perturbation approach. In the post-collision interval, $\psi_{1 c}(t, z)$ can be written as:

$$
\begin{aligned}
& \psi_{1 c}(t, z)=\left\{\frac{\eta_{1}}{\cosh \left(x_{1}\right)}-\Delta \eta_{12}^{(1)} \frac{\left[x_{1} \tanh \left(x_{1}\right)-1\right]}{\cosh \left(x_{1}\right)}+v_{12 c}(t, z)\right\} \\
& \times \exp \left\{i\left[\chi_{1}(t, z)+\Delta \alpha_{11}^{(0)}\right]\right\},
\end{aligned}
$$

where $x_{1}, \chi_{1}(t, z)$, and $\Delta \alpha_{11}^{(0)}$ were defined in the last paragraph of Sec. II B.

The time dependence of $\left|v_{12 b}(t, z)\right|$ and $\left|v_{12 c}(t, z)\right|$ at $z=z_{c}+2, z=z_{c}+5$, and $z=z_{c}+10$ is shown in Figs. 1 and 2 in the case where $\beta=10, \epsilon_{3}=0.02$, and $\eta_{1}=\eta_{2}=1$. In addition, Figs. 3 and 4 show the profiles of the total envelope of the electric field $\left|\psi_{1 b}(t, z)\right|$ and $\left|\psi_{1 c}(t, z)\right|$ obtained with the two versions of the perturbation theory at the same distances and for the same physical parameter values. As can be seen from Figs. 1 and 3, the radiation profiles $\left|v_{12 b}(t, z)\right|$ and $\left|v_{12 c}(t, z)\right|$ are very close at the three distances, and the pulse profiles $\left|\psi_{1 b}(t, z)\right|$ and $\left|\psi_{1 c}(t, z)\right|$ are also very close. In addition, it can be seen from Figs. 2 and 4 that the differences between the radiation and pulse profiles obtained with the two versions of the perturbation approach in the time interval $[-50,50]$ are of order $10^{-5}$. The tails of 

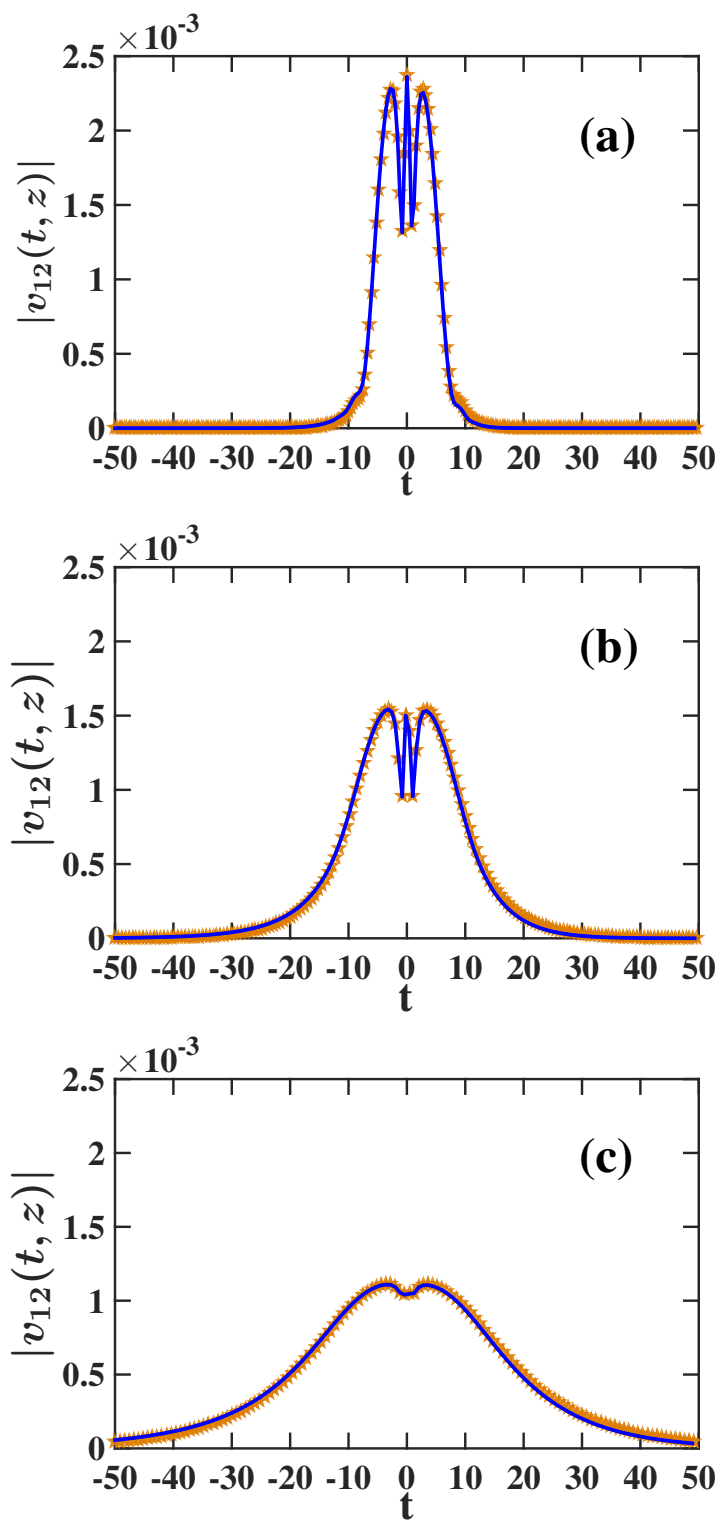

FIG. 1: The $t$ dependence of the collision-induced radiation profiles obtained with the basic and improved versions of the perturbation approach $\left|v_{12 b}(t, z)\right|$ and $\left|v_{12 c}(t, z)\right|$ at $z=z_{c}+2(\mathrm{a}), z=z_{c}+5$ (b), and $z=z_{c}+10(\mathrm{c})$. The parameter values are $\beta=10, \epsilon_{3}=0.02$, and $\eta_{1}=\eta_{2}=1$. The orange stars represent $\left|v_{12 b}(t, z)\right|$, as obtained with Eqs. (18) and (20). The solid blue line represents $\left|v_{12 c}(t, z)\right|$, as obtained with Eqs. 25) and (31).

the radiation and pulse profiles obtained with the basic perturbation approach are larger than the ones obtained with the improved approach for $t \gg 1$ and are smaller for $t \ll-1$. The magnitude of the differences between the two theoretical predictions can be explained by noting that the improved perturbative calculation incorporates effects of order $\epsilon_{3} / \beta^{2}$, 

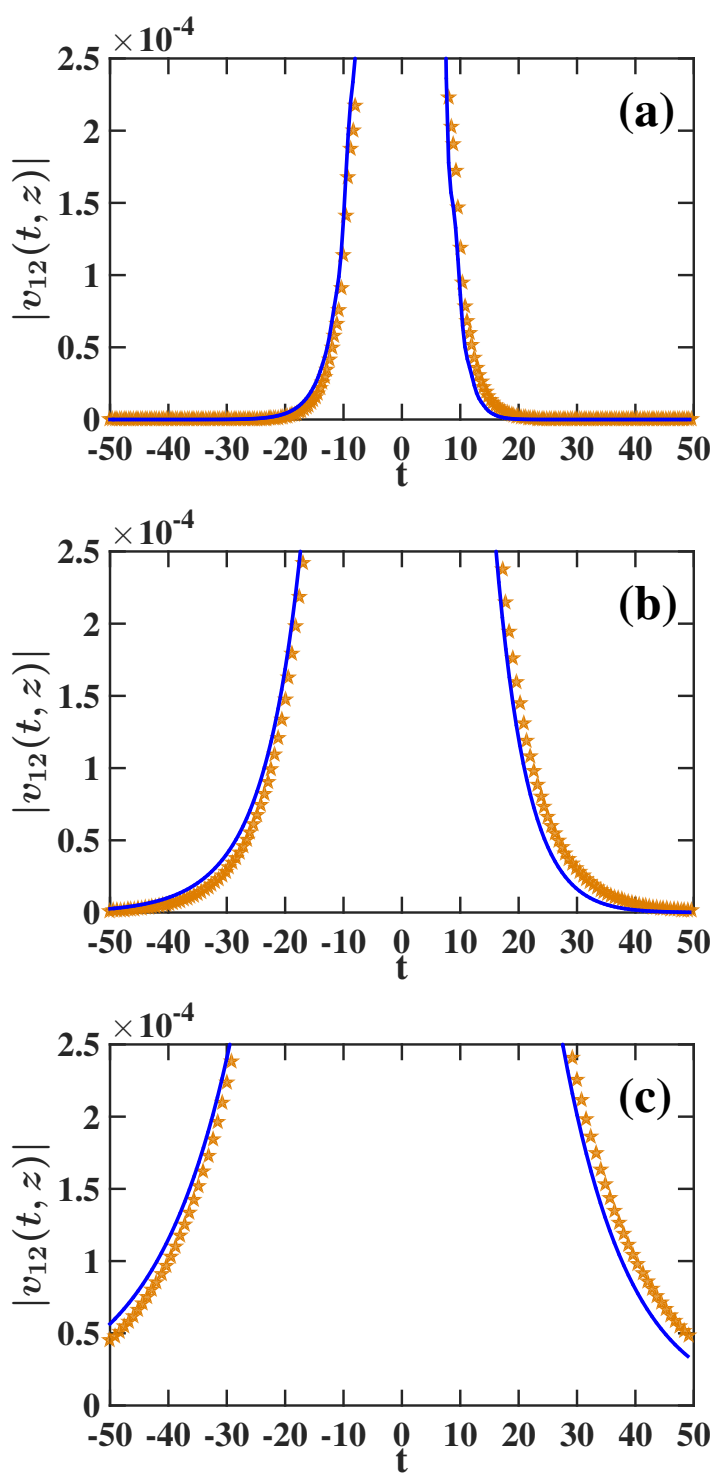

FIG. 2: Magnified versions of the graphs in Fig. 1 for small values of $\left|v_{12 b}(t, z)\right|$ and $\left|v_{12 c}(t, z)\right|$. The symbols, distances, and physical parameter values are the same as in Fig. 1 .

which are not taken into account in the basic perturbative calculation. For the parameter values used in the example, $\epsilon_{3} / \beta^{2}=2 \times 10^{-5}$, which is of the same order of magnitude as the differences between the two predictions for the radiation and pulse profiles that are observed in Figs. 2 and 4 . 

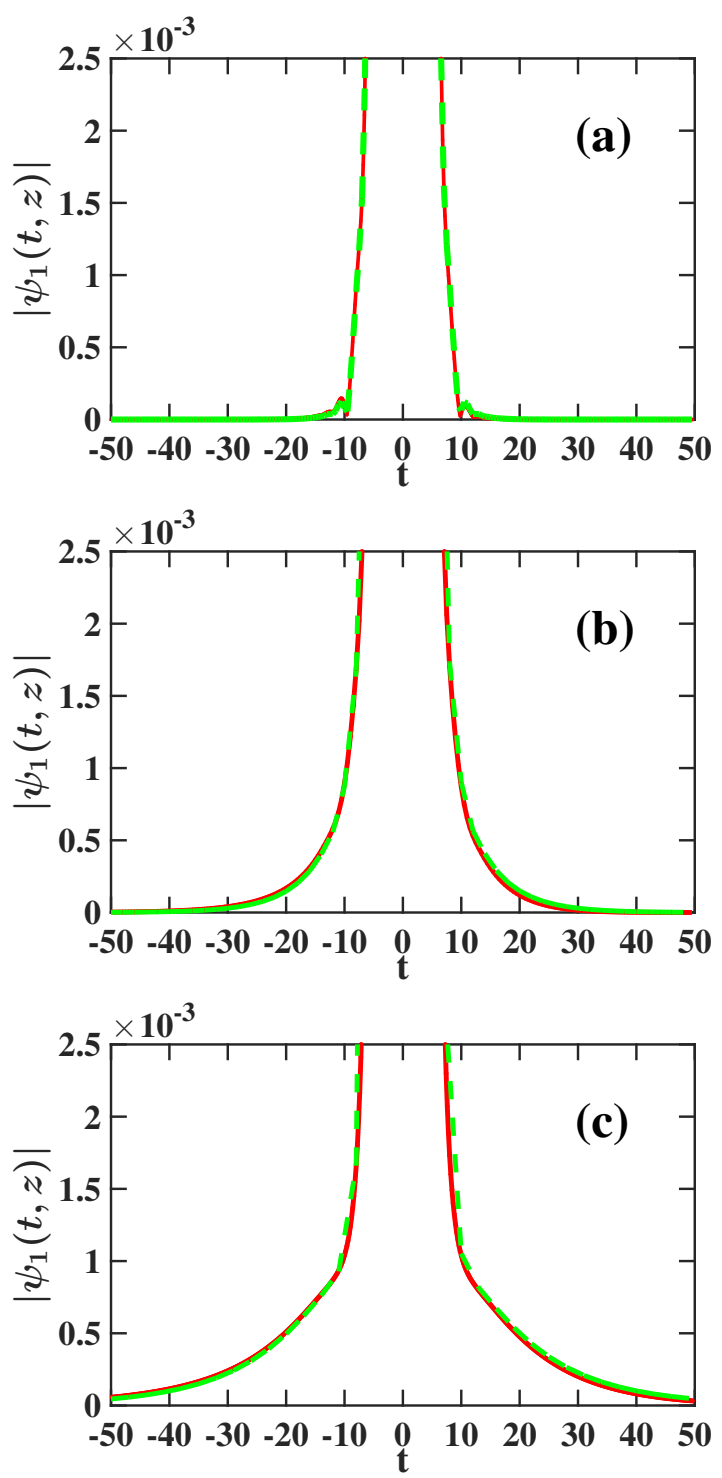

FIG. 3: The $t$ dependence of the pulse profiles obtained with the basic and improved versions of the perturbation approach $\left|\psi_{1 b}(t, z)\right|$ and $\left|\psi_{1 c}(t, z)\right|$ at $z=z_{c}+2(\mathrm{a}), z=z_{c}+5(\mathrm{~b})$, and $z=z_{c}+10$ (c). The parameter values are the same as in Fig. 1. The dashed green and solid red lines represent $\left|\psi_{1 b}(t, z)\right|$ and $\left|\psi_{1 c}(t, z)\right|$, respectively.

\section{Radiation dynamics in the equivalent single-pulse propagation problem}

In this subsection we provide a simpler treatment of the fast two-soliton collision in the presence of weak cubic loss that involves a single perturbed NLS equation. This simpler approach gives an equivalent description of radiation dynamics in order $\epsilon_{3} / \beta$, which is the leading order of the perturbation theory for radiation emission effects. Our reasons for 

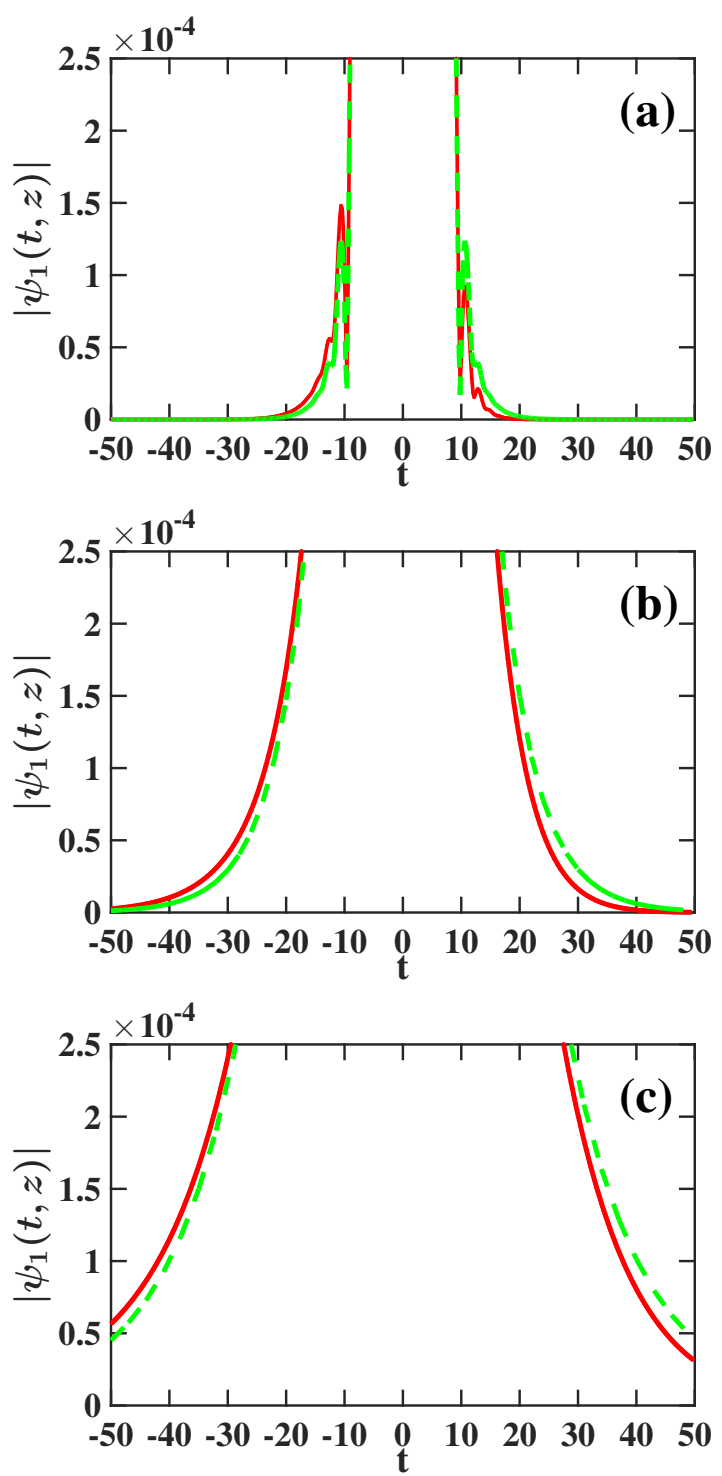

FIG. 4: Magnified versions of the graphs in Fig. 3 for small values of $\left|\psi_{1 b}(t, z)\right|$ and $\left|\psi_{1 c}(t, z)\right|$. The symbols, distances, and physical parameter values are the same as in Fig. 3 .

providing the simpler equivalent treatment of the collision problem are the following. (a) The simpler description helps in verifying the identification of the physical effects that are involved in radiation emission. In particular, by comparing the results of numerical simulations of the full coupled-NLS model (1) with results of the perturbed NLS equation for the equivalent single-pulse propagation problem we can determine if collision-induced changes in the shape of soliton 2 (distortion) are important for the dynamics of the radiation emitted by soliton 1 . In addition, we can determine if physical processes other than cubic loss, such as interpulse interaction due to Kerr nonlinearity, have an important effect on radiation dy- 
namics. (b) The single-pulse propagation problem can be formulated in a general manner, such that it describes propagation of soliton 1 in the presence of a generic fast and localized variation in the effective linear gain-loss coefficient. This generalization enables the calculation of radiation dynamics due to a wide class of fast and localized dissipative processes. The fast two-soliton collision discussed in Secs. IIA - IIC is just one example of these processes. (c) The perturbed NLS equation for the equivalent single-soliton propagation problem can be regarded as an intermediate model between the coupled-NLS equation (1) for the full single two-soliton collision problem and stochastic perturbed NLS models, which describe soliton propagation in multisequence nonlinear optical waveguide systems (see Refs. [16, 34, 35] for studies of the latter models). Therefore, by showing that the perturbed NLS equation for the equivalent single-soliton propagation problem accurately describes the dynamics of radiation in the full two-soliton collision problem, we provide strong evidence that the collision-induced radiation dynamics is accurately captured by stochastic perturbed NLS models for soliton propagation in multisequence nonlinear optical waveguide systems.

Consider the general version of the single-pulse propagation problem, in which the soliton propagates in the presence of second-order dispersion, Kerr nonlinearity, and a fast and localized variation in the effective linear gain-loss coefficient. The change in the linear gainloss coefficient can be due an actual change in the optical waveguide's properties or due to an external process, such as a fast collision between the soliton and another optical pulse. Thus, the propagation is described by the following perturbed NLS equation:

$$
i \partial_{z} \psi_{1}+\partial_{t}^{2} \psi_{1}+2\left|\psi_{1}\right|^{2} \psi_{1}=i \epsilon_{l} g_{l}(t, z) \psi_{1},
$$

where $0<\left|\epsilon_{l}\right| \ll 1$, and $g_{l}(t, z)$ is a real-valued function, which describes the fast and localized variation in the effective linear gain-loss coefficient. More specifically, $g_{l}(t, z)$ is of the form

$$
g_{l}(t, z)=g\left(x_{l}\right) \quad \text { with } \quad x_{l}=t-t_{l}+\bar{b} z,
$$

where $g\left(x_{l}\right)$ is sharply-peaked at $x_{l}=0, t_{l}$ and $\bar{b}$ are constants, and $\left|\bar{b}-2 \beta_{1}(0)\right| \gg 1$.

Let us obtain the expression for $g_{l}(t, z)$ for a fast two-soliton collision in the presence of weak cubic loss. For this purpose, we first note that the main effects of the collision on soliton 1 that are due to cubic loss, i.e., the collision-induced effects in order $\epsilon_{3} / \beta$, can be described by adding a term of the form $i \exp \left(i \chi_{1}\right) \partial_{z} \Phi_{12}^{(1)}$ to the unperturbed NLS equation 
for soliton 1. From Eq. (8) it follows that this term is of the form

$$
i \exp \left(i \chi_{1}\right) \partial_{z} \Phi_{12}^{(1)}=-\frac{2 i \epsilon_{3} \eta_{2}^{2}}{\cosh ^{2}\left(x_{2}\right)} \psi_{1}
$$

Therefore, the perturbed NLS equation for the equivalent single-soliton propagation problem is given by

$$
i \partial_{z} \psi_{1}+\partial_{t}^{2} \psi_{1}+2\left|\psi_{1}\right|^{2} \psi_{1}=-\frac{2 i \epsilon_{3} \eta_{2}^{2}}{\cosh ^{2}\left(x_{2}\right)} \psi_{1}
$$

Equating the right hand sides of Eqs. (36) and (33) we find that in a fast two-soliton collision in the presence of weak cubic loss $\epsilon_{l}=-\epsilon_{3}$ and $g_{l}(t, z)=2 \eta_{2}^{2} / \cosh ^{2}\left(x_{2}\right)$.

We now show that the equations for radiation dynamics obtained with the simpler perturbed NLS model (36) are identical to the equations obtained in Secs. II B and II C for the full two-soliton collision problem. For simplicity and without loss of generality, we take the initial frequencies as $\beta_{1}(0)=0$ and $\beta_{2}(0)=\beta$. We assume that $0<\epsilon_{3} \ll 1$ and $|\beta| \gg 1$ and look for a solution of Eq. (36) in the form

$$
\psi_{1}(t, z)=\psi_{s 1}(t, z)+\tilde{\phi}_{1}(t, z)
$$

where $\psi_{s 1}(t, z)$ is the single-soliton solution of the unperturbed NLS equation and $\tilde{\phi}_{1}(t, z)$ describes collision-induced changes to $\psi_{s 1}(t, z)$. We substitute the ansatz (37) into Eq. (36) and keep terms up to order $\epsilon_{3} / \beta$ in the equation. We obtain:

$$
i \partial_{z} \tilde{\phi}_{1}+\partial_{t}^{2} \tilde{\phi}_{1}+4\left|\psi_{s 1}\right|^{2} \tilde{\phi}_{1}+2 \psi_{s 1}^{2} \tilde{\phi}_{1}^{*}=-\frac{2 i \epsilon_{3} \eta_{2}^{2}}{\cosh ^{2}\left(x_{2}\right)} \psi_{s 1} .
$$

Next, we substitute $\psi_{s 1}(t, z)=\Psi_{1}\left(x_{1}\right) \exp \left(i \chi_{1}\right)$ and $\tilde{\phi}_{1}(t, z)=\tilde{\Phi}_{1}(t, z) \exp \left(i \chi_{1}\right)$ into Eq. (38). This substitution yields the following equation for $\tilde{\Phi}_{1}$ :

$$
i \partial_{z} \tilde{\Phi}_{1}+\left(\partial_{t}^{2}-\eta_{1}^{2}\right) \tilde{\Phi}_{1}+4 \Psi_{1}^{2} \tilde{\Phi}_{1}+2 \Psi_{1}^{2} \tilde{\Phi}_{1}^{*}=-\frac{2 i \epsilon_{3} \eta_{1} \eta_{2}^{2}}{\cosh \left(x_{1}\right) \cosh ^{2}\left(x_{2}\right)}
$$

Equation 39 is solved by expanding $\tilde{\Phi}_{1}$ in a perturbation series with respect to $\epsilon_{3}$ and $1 / \beta$ :

$$
\begin{aligned}
& \tilde{\Phi}_{1}(t, z)=\tilde{\Phi}_{11}^{(0)}(t, z)+\tilde{\Phi}_{11}^{(1)}(t, z)+\tilde{\Phi}_{12}^{(0)}(t, z) \\
& +\tilde{\Phi}_{12}^{(1)}(t, z)+\ldots,
\end{aligned}
$$

where the subscripts and superscript notations are similar to the ones in Eq. (5). 
The equation describing the collision-induced effects in order $\epsilon_{3} / \beta$, which is the leading order, is:

$$
\partial_{z} \tilde{\Phi}_{12}^{(1)}=-\frac{2 \epsilon_{3} \eta_{1} \eta_{2}^{2}}{\cosh \left(x_{1}\right) \cosh ^{2}\left(x_{2}\right)} .
$$

This equation is identical to Eq. (8), which was obtained with the basic version of the perturbative calculation for the full two-soliton collision problem. As a result, the equations for radiation dynamics obtained in the equivalent single-soliton propagation problem in order $\epsilon_{3} / \beta$ are identical to the equations obtained in Sec. II B with the basic version of the perturbation approach for the full two-soliton collision problem.

We can also obtain an improved approximation for radiation dynamics by taking into account the effects of propagation of radiation in the collision interval, which are described by the $O\left(\epsilon_{3} / \beta^{2}\right)$ terms $\left(\partial_{t}^{2}-\eta_{1}^{2}\right) \tilde{\Phi}_{1}, 4 \Psi_{1}^{2} \tilde{\Phi}_{1}$, and $2 \Psi_{1}^{2} \tilde{\Phi}_{1}$ in Eq. 39. For this purpose, we denote by $\tilde{\Phi}_{12 c}^{(1)}$ the part of $\tilde{\Phi}_{1}$ that describes the collision effects in order $\epsilon_{3} / \beta$ and the effects of propagation of radiation in the collision interval. $\tilde{\Phi}_{12 c}^{(1)}$ satisfies the following equation:

$$
\partial_{z} \tilde{\Phi}_{12 c}^{(1)}-i\left[\left(\partial_{t}^{2}-\eta_{1}^{2}\right) \tilde{\Phi}_{12 c}^{(1)}+4 \Psi_{1}^{2} \tilde{\Phi}_{12 c}^{(1)}+2 \Psi_{1}^{2} \tilde{\Phi}_{12 c}^{(1) *}\right]=-\frac{2 \epsilon_{3} \eta_{1} \eta_{2}^{2}}{\cosh \left(x_{1}\right) \cosh ^{2}\left(x_{2}\right)} .
$$

Equation (42) is identical to Eq. (22) that was obtained with the improved perturbative calculation for the full two-soliton collision problem. Therefore, the equations for radiation dynamics obtained with the improved approximation in the equivalent single-soliton propagation problem are identical to the equations obtained in Sec. II C with the improved perturbation approach for the full two-soliton collision problem.

We point out that the same perturbation approaches, which were described in Secs. II B and IIC, can be used for analyzing radiation dynamics in the general problem of singlesoliton propagation in the presence of a fast and localized variation in the linear gain-loss coefficient. Indeed, substituting the ansatz (37) into the general perturbed NLS model (33), we arrive at the following equations for $\tilde{\Phi}_{12}^{(1)}$ and $\tilde{\Phi}_{12 c}^{(1)}$ in the general propagation problem:

$$
\partial_{z} \tilde{\Phi}_{12}^{(1)}=\frac{\epsilon_{l} \eta_{1} g\left(x_{l}\right)}{\cosh \left(x_{1}\right)}
$$

and

$$
\partial_{z} \tilde{\Phi}_{12 c}^{(1)}-i\left[\left(\partial_{t}^{2}-\eta_{1}^{2}\right) \tilde{\Phi}_{12 c}^{(1)}+4 \Psi_{1}^{2} \tilde{\Phi}_{12 c}^{(1)}+2 \Psi_{1}^{2} \tilde{\Phi}_{12 c}^{(1) *}\right]=\frac{\epsilon_{l} \eta_{1} g\left(x_{l}\right)}{\cosh \left(x_{1}\right)} .
$$

Equations (43) and (44) have a form similar to Eqs. (8) and (22) that were analyzed in Secs. II B and IIC, respectively. Therefore, the equations for radiation dynamics in the general 
single-soliton propagation problem, described by Eq. (33), can be obtained by employing the perturbation approaches of Secs. II B and II C.

\section{NUMERICAL SIMULATIONS}

\section{A. Introduction}

The analytic predictions for radiation dynamics obtained in Secs. IIB - IID are based on several simplifying approximations. In particular, the perturbative calculations in these sections only take into account the $O\left(\epsilon_{3} / \beta\right)$ effects of cubic loss and the $O\left(\epsilon_{3} / \beta^{2}\right)$ effects associated with propagation of radiation in the collision interval. This means that the perturbative calculations neglect the distortion of soliton 2 and the effect of interpulse interaction due to Kerr nonlinearity on radiation dynamics. Since the validity and accuracy of these approximations depend on the values of the physical parameters, it is important to check the analytic predictions obtained in Secs. IIB - IID by numerical simulations with the full coupled-NLS model (1).

To gain further insight into the physical processes that determine radiation dynamics and into the reasons for differences between the analytic predictions and results of simulations with Eq. (1), we carry out numerical simulations with four additional simpler propagation models. The first additional model is a modified version of the perturbed NLS equation (36), which takes into account collision-induced radiation emission due to both cubic loss and Kerr nonlinearity, and position shifts due to both Kerr nonlinearity and the collisioninduced frequency shift. As we show in Appendix B, this model has the form:

$$
i \partial_{z} \psi_{1}+\partial_{t}^{2} \psi_{1}+2\left|\psi_{1}\right|^{2} \psi_{1}=-\frac{2 i \epsilon_{3} \eta_{2}^{2}}{\cosh ^{2}\left(x_{2}\right)} \psi_{1}-\frac{4 \eta_{2}^{2}}{\cosh ^{2}\left(x_{2}\right)} \psi_{1}-i C_{1}(z) \partial_{t} \psi_{1},
$$

where $C_{1}(z)$ is given by

$$
C_{1}(z)= \begin{cases}0 & \text { for } z<z_{c} \\ \frac{40 \epsilon_{3} \eta_{1}^{2} \eta_{2}}{3|\beta| \beta} & \text { for } z \geq z_{c}\end{cases}
$$

The second term on the right hand side of Eq. 45) describes radiation emission and the collision-induced position shift due to Kerr nonlinearity. The third term on the right hand side of Eq. (45) describes the position shift caused by the collision-induced frequency shift. 
The most important difference between Eq. (45) and the full coupled-NLS model (1) is that Eq. 45 neglects the distortion of soliton 2 and its effects on the collision.

The second simplified NLS model is a variation of Eq. (45), in which the second term on the right hand side is replaced by a term that describes only the Kerr-induced position shift and neglects the Kerr-induced radiation emission in the collision. In Appendix B, we show that this model takes the form:

$$
i \partial_{z} \psi_{1}+\partial_{t}^{2} \psi_{1}+2\left|\psi_{1}\right|^{2} \psi_{1}=-\frac{2 i \epsilon_{3} \eta_{2}^{2}}{\cosh ^{2}\left(x_{2}\right)} \psi_{1}-i C_{2}(z) \partial_{t} \psi_{1}-i C_{1}(z) \partial_{t} \psi_{1},
$$

where $C_{2}(z)$ is given by

$$
C_{2}(z)= \begin{cases}0 & \text { for } z<z_{c}-\frac{1}{2|\beta|} \\ \frac{4 \eta_{2}}{|\beta|} & \text { for } z_{c}-\frac{1}{2|\beta|} \leq z \leq z_{c}+\frac{1}{2|\beta|} \\ 0 & \text { for } z>z_{c}+\frac{1}{2|\beta|}\end{cases}
$$

Equation (48) neglects distortion of soliton 2 and Kerr-induced radiation emission in the collision. The latter two effects are taken into account in the full coupled-NLS model.

The third simplified model is the following coupled-NLS model, which takes into account distortion of soliton 2 in describing the effects of cubic loss on the collision, but neglects distortion of soliton 2 in describing the effects of Kerr nonlinearity on the collision. This model also takes into account the position shift due to the collision-induced frequency shift. As shown in Appendix B, this third simplified propagation model is given by:

$$
\begin{aligned}
& i \partial_{z} \psi_{1}+\partial_{t}^{2} \psi_{1}+2\left|\psi_{1}\right|^{2} \psi_{1}=-2 i \epsilon_{3}\left|\psi_{2}\right|^{2} \psi_{1}-\frac{4 \eta_{2}^{2}}{\cosh ^{2}\left(x_{2}\right)} \psi_{1}-i C_{1}(z) \partial_{t} \psi_{1}, \\
& i \partial_{z} \psi_{2}+\partial_{t}^{2} \psi_{2}+2\left|\psi_{2}\right|^{2} \psi_{2}=-2 i \epsilon_{3}\left|\psi_{1}\right|^{2} \psi_{2}-\frac{4 \eta_{1}^{2}}{\cosh ^{2}\left(x_{1}\right)} \psi_{2}-i C_{3}(z) \partial_{t} \psi_{2},
\end{aligned}
$$

where $C_{3}(z)$ is

$$
C_{3}(z)= \begin{cases}0 & \text { for } z<z_{c} \\ \frac{-40 \epsilon_{3} \eta_{1} \eta_{2}^{2}}{3|\beta| \beta} & \text { for } z \geq z_{c}\end{cases}
$$

The main difference between Eq. (49) and the full coupled-NLS model (1) is that Eq. (49) neglects the distortion of soliton 2 in describing the effects of Kerr nonlinearity on the collision.

The fourth simplified model is a variation of the coupled-NLS model (49), in which the second terms on the right hand sides are replaced by terms that describe only the Kerrinduced position shift and neglect the Kerr-induced radiation emission. In Appendix B, we 
show that this model has the form

$$
\begin{aligned}
& i \partial_{z} \psi_{1}+\partial_{t}^{2} \psi_{1}+2\left|\psi_{1}\right|^{2} \psi_{1}=-2 i \epsilon_{3}\left|\psi_{2}\right|^{2} \psi_{1}-i C_{2}(z) \partial_{t} \psi_{1}-i C_{1}(z) \partial_{t} \psi_{1} \\
& i \partial_{z} \psi_{2}+\partial_{t}^{2} \psi_{2}+2\left|\psi_{2}\right|^{2} \psi_{2}=-2 i \epsilon_{3}\left|\psi_{1}\right|^{2} \psi_{2}-i C_{4}(z) \partial_{t} \psi_{2}-i C_{3}(z) \partial_{t} \psi_{2}
\end{aligned}
$$

where

$$
C_{4}(z)= \begin{cases}0 & \text { for } z<z_{c}-\frac{1}{2|\beta|} \\ -\frac{4 \eta_{1}}{|\beta|} & \text { for } z_{c}-\frac{1}{2|\beta|} \leq z \leq z_{c}+\frac{1}{2|\beta|} \\ 0 & \text { for } z>z_{c}+\frac{1}{2|\beta|}\end{cases}
$$

\section{B. Description and discussion of simulations results}

Equations (1), 45), 47), 49, and (51) are numerically integrated on a time domain

$\left[t_{\min }, t_{\max }\right]=[-3200,3200]$ using the split-step method with periodic boundary conditions [7, 62. The large temporal domain is chosen such that the values of $\left|\psi_{1}(t, z)\right|$ and $\left|\psi_{2}(t, z)\right|$ at and near the boundaries are negligible throughout the simulation. As a result, potential artificial effects due to the radiation leaving the computational domain at one boundary and reentering it at another boundary are also negligible. The $t$-step and $z$-step of the numerical scheme are taken as $\Delta t=0.065$ and $\Delta z=0.0002$. These values ensure stability of the numerical scheme and provide sufficient accuracy for capturing the radiation dynamics.

For concreteness, we present the results of the simulations for two sets of values of $\epsilon_{3}$ and $\beta$ : (1) $\epsilon_{3}=0.02$ and $\beta=20 ;(2) \epsilon_{3}=0.02$ and $\beta=10$. This choice enables investigation of the dependence of the radiation dynamics on the frequency difference parameter $\beta$. More specifically, the results obtained for $\epsilon_{3}=0.02$ and $\beta=20$ are representative for large $\beta$ values, while the results obtained for $\epsilon_{3}=0.02$ and $\beta=10$ are representative for intermediate $\beta$ values. The initial condition for the simulations with Eqs. (1), (49), and (51) consists of two NLS solitons of the form (2) with frequencies $\beta_{1}(0)=0$ and $\beta_{2}(0)=\beta=20$ or 10 . The initial amplitudes and phases of the solitons are $\eta_{1}(0)=\eta_{2}(0)=1$ and $\alpha_{1}(0)=\alpha_{2}(0)=0$. The initial positions and the final propagation distance are $y_{1}(0)=0, y_{2}(0)=20$, and $z_{f}=10$. For these values, the two solitons are well-separated before and after the collision. In addition, the collision distance is $z_{c}=0.5$ for $\beta=20$ and $z_{c}=1.0$ for $\beta=10$. The initial condition for the simulations with Eqs. (45) and 47) is an NLS soliton of the form (2) with $\beta_{1}(0)=0, \eta_{1}(0)=1, y_{1}(0)=0$, and $\alpha_{1}(0)=0$. The parameters $\beta_{2}, \eta_{2}$, and $y_{2}$ in 
the equation are taken as $\beta_{2}=20$ or $10, \eta_{2}=1$, and $y_{2}=20$.

Figure 5 shows the $t$ dependence of the pulse profile $\left|\psi_{1}(t, z)\right|$ obtained in the numerical simulation with Eq. (1) for $\epsilon_{3}=0.02$ and $\beta=20$ at $z=z_{c}+2, z=z_{c}+5$, and $z=z_{c}+10$. Also shown are the predictions of the simple and improved versions of the perturbation approach $\left|\psi_{1 b}(t, z)\right|$ and $\left|\psi_{1 c}(t, z)\right|$, and the radiation profile $\left|v_{12 c}(t, z)\right|$ obtained with the improved perturbation approach. Figure 6 shows magnified versions of the graphs in Fig. 5 for small $\left|\psi_{1}(t, z)\right|$ values. We observe very good agreement between the predictions of the two perturbation approaches and the numerical simulation's result at all three distances. To further quantify the accuracy of the two predictions of the perturbation theory, we calculate the deviations ||$\psi_{1}^{(\text {num })}(t, z)|-| \psi_{1 b}(t, z) \|$ and ||$\psi_{1}^{(\text {num })}(t, z)|-| \psi_{1 c}(t, z)||$, where $\psi_{1}^{(\text {num })}(t, z)$ is the envelope of the electric field obtained in the simulation with Eq. (1). We find that the accuracies of the two predictions are comparable. In addition, the prediction of the improved perturbation approach for the radiative tails is more accurate at short distances, while the prediction of the simple perturbation approach is more accurate at long distances. For example, at $z=z_{c}+2$, we find that the improved theory's prediction is more accurate than the simple theory's prediction in the intervals $-50 \leq t<-10.77$, and $21.44<t \leq 50.0$ [63]. In contrast, at $z=z_{c}+10$, the simple theory's prediction is more accurate for $-45.84<t<-6.28$ and for $6.54<t \leq 50$, while the improved theory's prediction is more accurate for $-50 \leq t<-45.84$ 64. Moreover, we find that the accuracies of both predictions of the perturbation theory for the radiative tails increase with increasing distance. More specifically, for $|t|>6$, the deviations ||$\psi_{1}^{(\text {num })}(t, z)|-| \psi_{1 b}(t, z) \|$ and $\left\|\psi_{1}^{(\text {num })}(t, z)|-| \psi_{1 c}(t, z)\right\|$ are smaller than $4.77 \times 10^{-5}$ and $5.21 \times 10^{-5}$ at $z=z_{c}+2$, and are smaller than $1.59 \times 10^{-5}$ and $1.86 \times 10^{-5}$ at $z=z_{c}+10$. Thus, our numerical simulation with $\epsilon_{3}=0.02$ and $\beta=20$ validate the predictions of both perturbation approaches with high accuracy.

It is helpful to compare the predictions of the perturbation theory with results of numerical simulations with the four simplified NLS models (45), (47), (49), and (51). Figure 7 shows the pulse profile $\left|\psi_{1}(t, z)\right|$ obtained by the improved perturbation approach and by numerical solution of Eqs. (45), (47), (49), and (51) at $z=z_{c}+2, z=z_{c}+5$, and $z=z_{c}+10$. Figure 8 shows magnified versions of the graphs in Fig. 7 for small $\left|\psi_{1}(t, z)\right|$ values. We observe very good agreement between the improved perturbation theory's prediction and the results of the four simplified NLS models for all three distances. We recall that the 

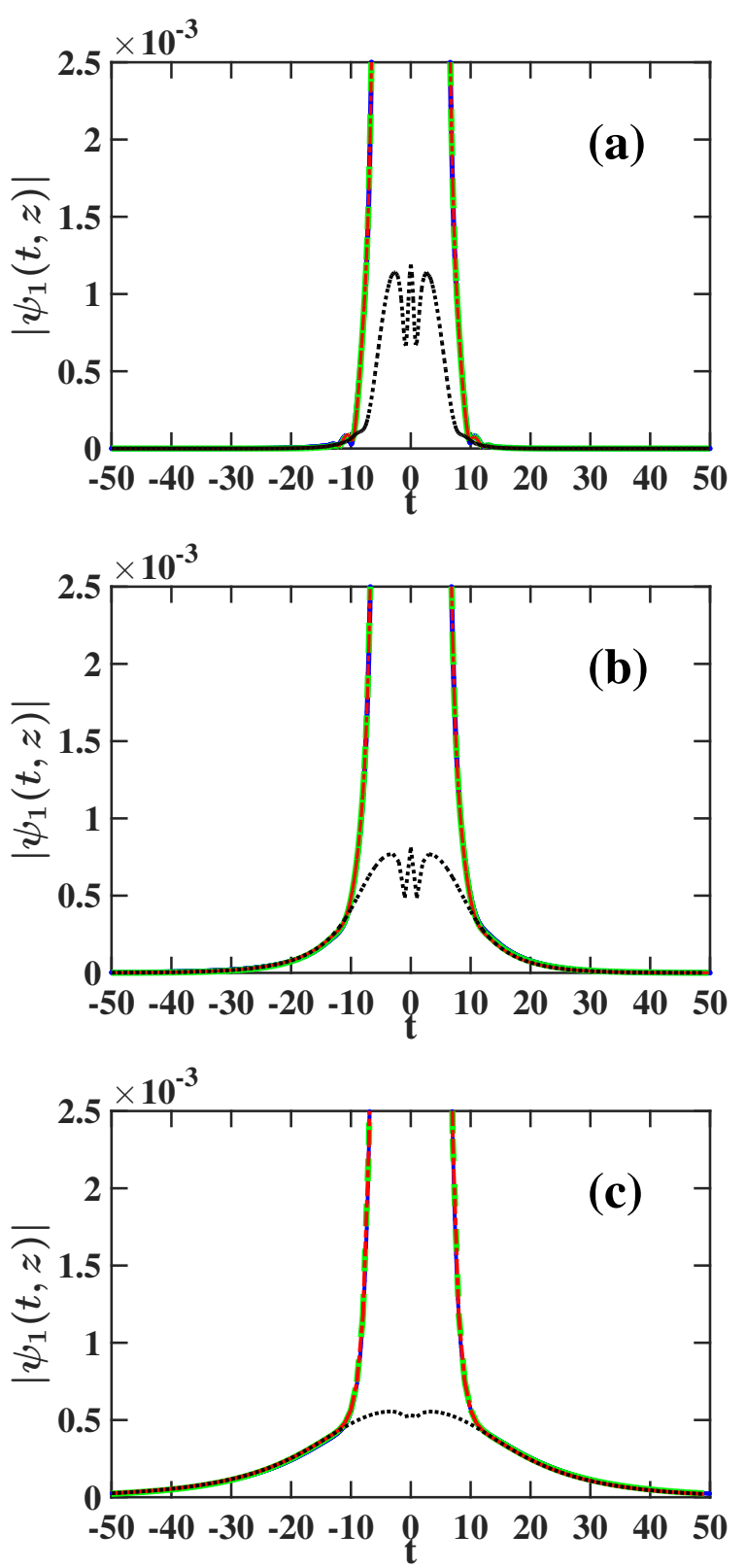

FIG. 5: A comparison between the perturbation theory's predictions and the result of numerical simulation with Eq. (1) for the $t$ dependence of the pulse profile $\left|\psi_{1}(t, z)\right|$. The physical parameter values are $\epsilon_{3}=0.02$ and $\beta=20$ and the distances are $z=z_{c}+2$ in (a), $z=z_{c}+5$ in (b), and $z=z_{c}+10$ in (c). The solid blue curve represents the result obtained by numerical solution of Eq. (1). The dashed green and dashed-dotted red curves correspond to the predictions of the basic and improved versions of the perturbation approach $\left|\psi_{1 b}(t, z)\right|$ and $\left|\psi_{1 c}(t, z)\right|$, respectively. The dotted black curve corresponds to the radiation profile $\left|v_{12 c}(t, z)\right|$ obtained with the improved perturbation procedure. 

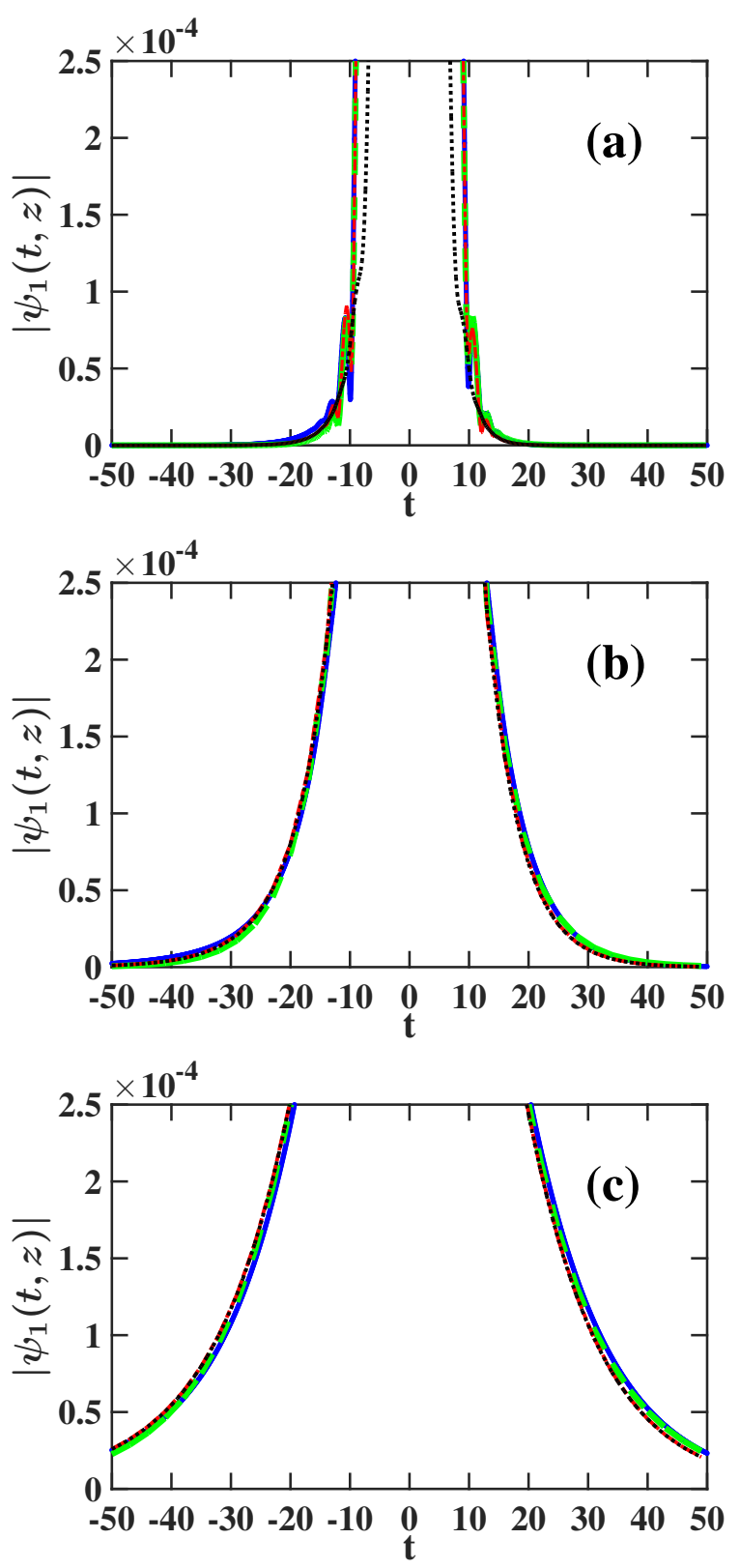

FIG. 6: Magnified versions of the graphs in Fig. 5 for small $\left|\psi_{1}(t, z)\right|$ values. The symbols are the same as in Fig. 5 .

perturbation theory and the models (47) and (51) neglect the effects of Kerr-induced interpulse interaction on radiation dynamics, but that these effects are taken into account in the models (45) and (49). Therefore, the comparison presented in Figs. 7 and 8 indicates that the effects of interpulse interaction due to Kerr nonlinearity on the collision-induced radiation dynamics are unimportant for $\epsilon_{3}=0.02$ and $\beta=20$.

To gain further insight into the physical processes that govern radiation dynamics in the 

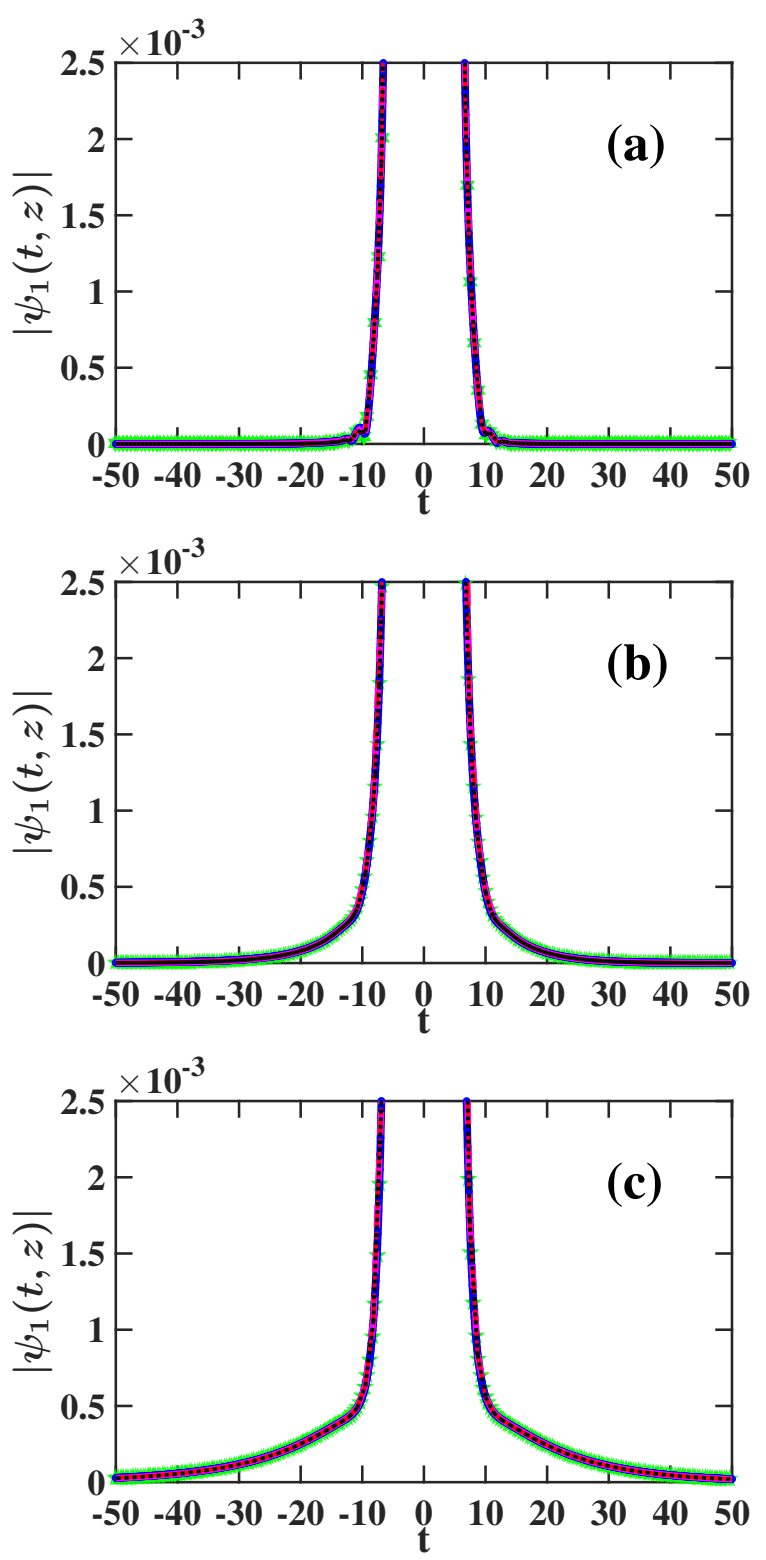

FIG. 7: A comparison between the $t$ dependences of the pulse profile $\left|\psi_{1}(t, z)\right|$ obtained by the improved perturbation approach and the pulse profiles obtained in numerical simulations with the four simplified NLS models (45), (47), (49), and (51). The parameter values are $\epsilon_{3}=0.02$ and $\beta=20$ and the distances are $z=z_{c}+2$ in (a), $z=z_{c}+5$ in (b), and $z=z_{c}+10$ in (c). The green stars represent the prediction of the improved perturbation approach. The solid-blue, dashed magenta, dashed-dotted red, and dotted black curves correspond to the pulse profiles obtained by numerical solution of Eqs. (45), 477, (49), and (51). 

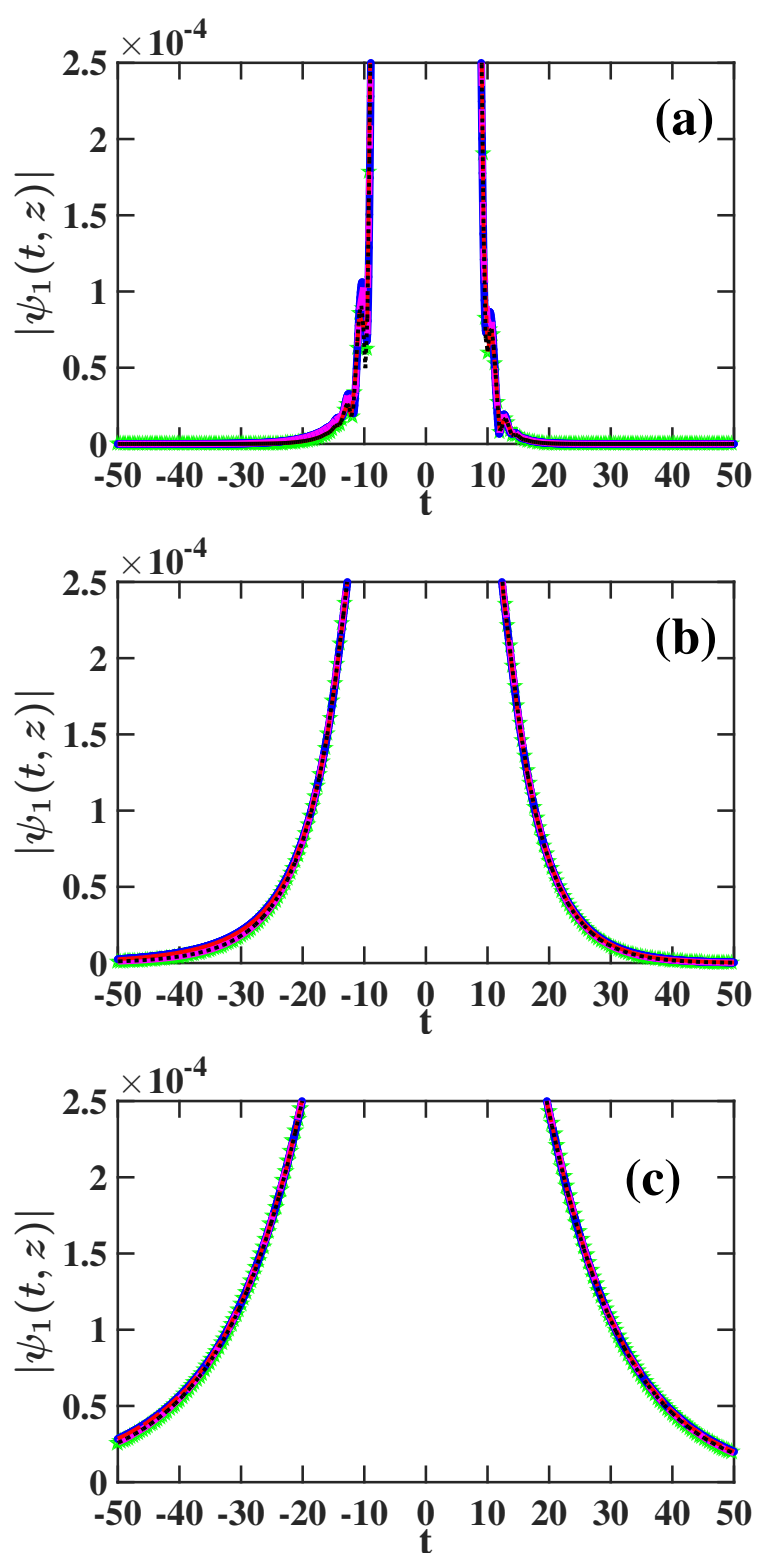

FIG. 8: Magnified versions of the graphs in Fig. 7 for small $\left|\psi_{1}(t, z)\right|$ values. The symbols are the same as in Fig. 7 .

collision we compare the pulse profile $\left|\psi_{1}^{(\text {num })}(t, z)\right|$ obtained in simulations with the full coupled-NLS model 11 with the pulse profiles $\left|\psi_{1}^{(\text {num }, s)}(t, z)\right|$ and $\left|\psi_{1}^{(\text {num }, p)}(t, z)\right|$ obtained in simulations with the two simplified NLS models (45) and (49), respectively. Figure 9 shows the comparison of the pulse profiles obtained in numerical simulations with Eqs. (1), (45), and (49) at $z=z_{c}+2, z=z_{c}+5$, and $z=z_{c}+10$. Figure 10 shows magnified versions of the graphs in Fig. 9 for small $\left|\psi_{1}(t, z)\right|$ values. We observe that the pulse 
profiles obtained with the simplified models (45) and (49) are very close to each other at the three distances. Furthermore, as seen in Figs. 9 and 10 , the pulse profiles obtained in numerical simulations with the two simplified models are in very good agreement with the result obtained in simulations with the full coupled-NLS model (1). More specifically, the deviations ||$\psi_{1}^{(\text {num })}(t, z)|-| \psi_{1}^{(\text {num }, s)}(t, z)||$ and ||$\psi_{1}^{(\text {num })}(t, z)|-| \psi_{1}^{(\text {num }, p)}(t, z) \|$ are smaller than $1.87 \times 10^{-4}$ and $1.95 \times 10^{-4}$ for all $t$ values at $z=z_{c}+2$, and are smaller than $7.57 \times 10^{-4}$ and $7.48 \times 10^{-4}$ for all $t$ values at $z=z_{c}+10$. Moreover, the deviations for $|t|>6$ are both smaller than $1.03 \times 10^{-4}$ at $z=z_{c}+2$, and are smaller than $5.45 \times 10^{-5}$ and $5.31 \times 10^{-5}$ at $z=z_{c}+10$. Thus, the agreement between the results of the two simplified NLS models for the pulse tails, where radiation is dominant, and the result of the full coupled-NLS model improves with increasing propagation distance. Recall that distortion of soliton 2 is completely neglected in Eq. (45). Therefore, the good agreement between the results obtained with Eqs. (45) and (1) means that for $\epsilon_{3}=0.02$ and $\beta=20$, distortion of soliton 2 does not play an important role in the collision-induced radiation dynamics of soliton 1. Additionally, as seen in Figs. 7 and 8 , for $\beta=20$ and $\epsilon_{3}=0.02$, the results of numerical simulations with Eqs. (45) and (49) are in very good agreement with the results obtained with the simplified models (47) and (51), which neglect radiation emission due to Kerr-induced interpulse interaction. Therefore, based on the comparison in Figs. 7- 10 we conclude that the contribution of Kerr-induced interpulse interaction to radiation dynamics is very small for $\beta=20$ and $\epsilon_{3}=0.02$. It follows that in this case, we can use the two simplified NLS models (47) and (51) to analyze radiation dynamics with very good accuracy.

We now turn to analyze the results of numerical simulations with $\epsilon_{3}=0.02$ and $\beta=$ 10 in comparison with the predictions of the perturbation theory. Figure 11 shows the $t$ dependence of the pulse profile obtained in the simulation with Eq. (1) $\left|\psi_{1}^{(\text {num })}(t, z)\right|$ at $z=z_{c}+2, z=z_{c}+5$, and $z=z_{c}+10$. The predictions of the simple and improved perturbation approaches $\left|\psi_{1 b}(t, z)\right|$ and $\left|\psi_{1 c}(t, z)\right|$, and the radiation profile obtained with the improved perturbation approach $\left|v_{12 c}(t, z)\right|$ are also shown. Figure 12 shows magnified versions of the graphs in Fig. 11 for small $\left|\psi_{1}(t, z)\right|$ values. We observe that the tails of the pulse profile obtained by the simulation with Eq. (1) for $\epsilon_{3}=0.02$ and $\beta=10$ are larger than the tails obtained with Eq. (1) for $\epsilon_{3}=0.02$ and $\beta=20$. This finding coincides with the expected increase in the strength of the collision-induced effects with decreasing value of $\beta$, see, e.g., Refs. [24, 29]. Furthermore, we find good agreement between 

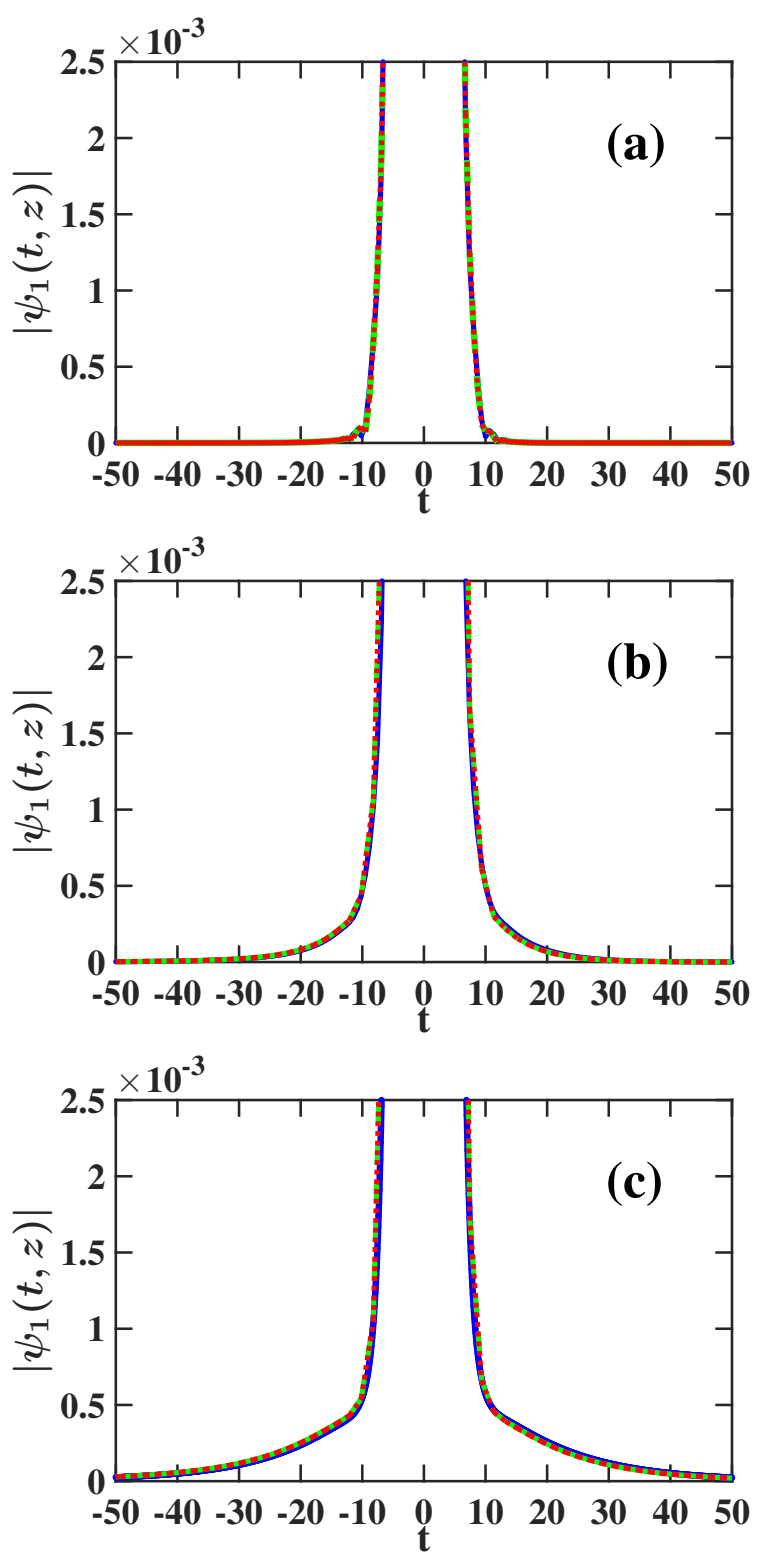

FIG. 9: A comparison between the $t$ dependences of the pulse profile $\left|\psi_{1}(t, z)\right|$ obtained in numerical simulations with the three perturbed NLS models (1), 45), and (49). The physical parameter values are $\epsilon_{3}=0.02$ and $\beta=20$ and the distances are $z=z_{c}+2$ in (a), $z=z_{c}+5$ in (b), and $z=z_{c}+10$ in (c). The solid blue, dashed green and dotted red curves represent $\left|\psi_{1}(t, z)\right|$ obtained by numerical solution of Eqs. (1), (45), and (49), respectively.

the numerical simulation's result and the perturbation theory predictions. However, the agreement is not as good as the one obtained for $\epsilon_{3}=0.02$ and $\beta=20$. In particular, the deviations of the numerically obtained pulse shape from the perturbation theory predictions 

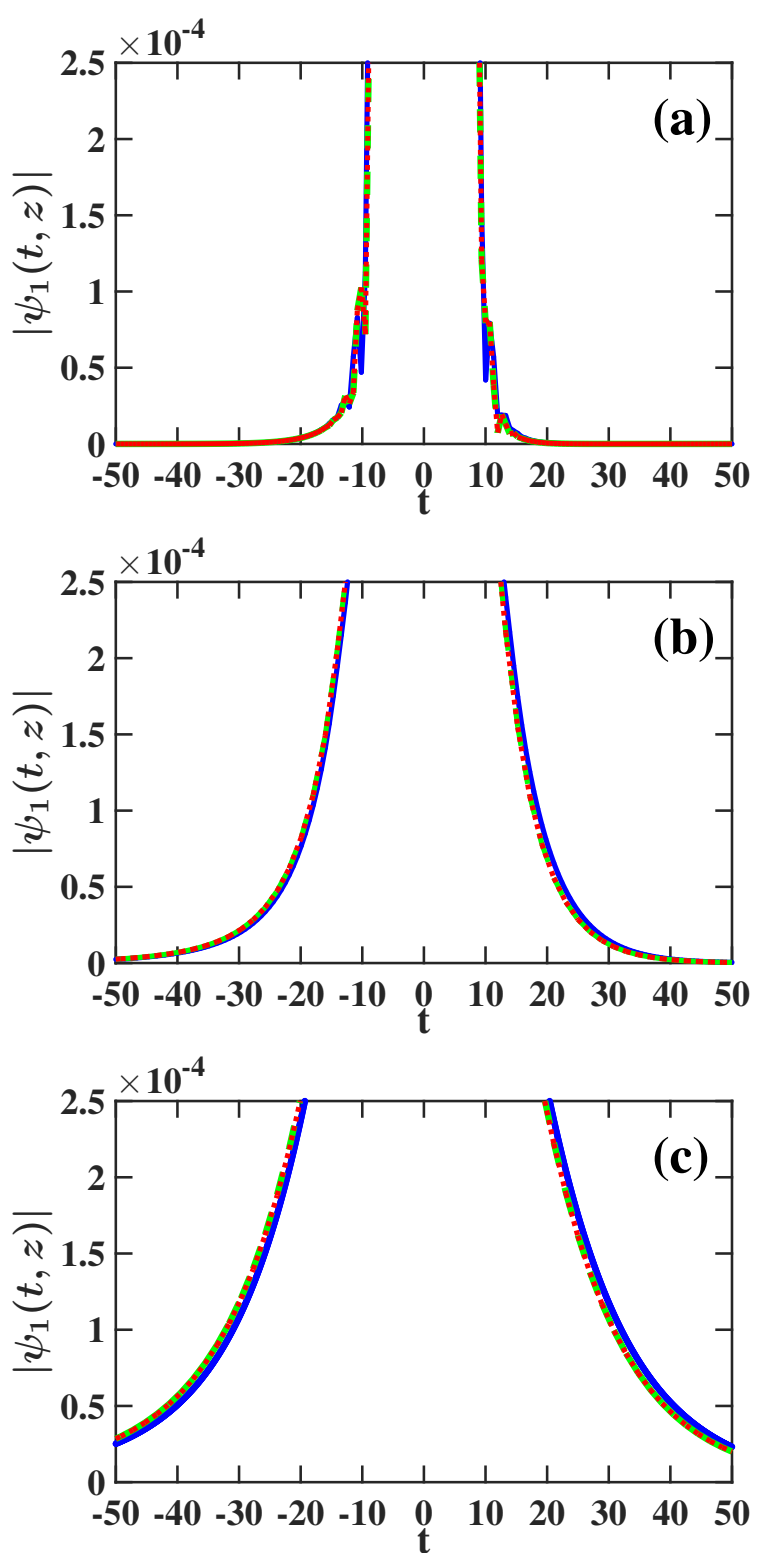

FIG. 10: Magnified versions of the graphs in Fig. 9 for small $\left|\psi_{1}(t, z)\right|$ values. The symbols are the same as in Fig. 9

are more significant for negative $t$ values and are smaller for positive $t$ values (see Fig. 12). For example, the deviations ||$\psi_{1}^{(\text {num })}(t, z)|-| \psi_{1 b}(t, z) \|$ and ||$\psi_{1}^{(\text {num })}(t, z)|-| \psi_{1 c}(t, z) \|$ at $z=z_{c}+5$ are larger than $1.0 \times 10^{-4}$ in the intervals $-30.19<t<-16.08$ and $-26.47<$ $t<-20.18$, respectively. In contrast, at the same distance these deviations are smaller than $1.0 \times 10^{-4}$ for all $t$ such that $t>9.76$ and $t>9.63$, respectively. We also observe that the prediction of the simple perturbation approach for the pulse tails is in better agreement with the simulation's result compared with the improved version for positive $t$ values. The 
improved perturbation approach is in slightly better agreement with the numerical result for negative $t$ values. Additionally, similar to the situation for $\epsilon_{3}=0.02$ and $\beta=20$, the agreement between the two perturbative predictions and the numerical simulation's result for the pulse tails improves with increasing distance. For example, for $|t|>6$, the deviations $\left\|\psi_{1}^{(\text {num })}(t, z)|-| \psi_{1 b}(t, z)\right\|$ and ||$\psi_{1}^{(\text {num })}(t, z)|-| \psi_{1 c}(t, z) \|$ are smaller than $3.81 \times 10^{-4}$ and $3.90 \times 10^{-4}$ at $z=z_{c}+2$, and are smaller than $1.20 \times 10^{-4}$ and $1.14 \times 10^{-4}$ at $z=z_{c}+10$.

Further insight into the collision-induced radiation dynamics can be gained by comparing the predictions of the perturbation theory with results of numerical simulations with the four simplified NLS models (45), 47), 49), and (51). Figure 13 shows the pulse profile $\left|\psi_{1}(t, z)\right|$ obtained by the improved perturbation approach and by numerical solution of Eqs. (45), (47), (49), and (51) at $z=z_{c}+2, z=z_{c}+5$, and $z=z_{c}+10$. Figure 14 shows magnified versions of the graphs in Fig. 13 for small $\left|\psi_{1}(t, z)\right|$ values. We observe very good agreement between the perturbation theory's prediction and the results of numerical simulations with the NLS models (47) and (51). There is also very good agreement between the results of the NLS models 45 and (49). In contrast, the values of $\left|\psi_{1}(t, z)\right|$ at the pulse tails obtained with Eqs. (45) and (49) are noticeably larger than the $\left|\psi_{1}(t, z)\right|$ values at the pulse tails obtained with the perturbation theory and with the NLS models (47) and (51) (see Fig. 14. These deviations are more significant for negative $t$ values, and they decrease with increasing propagation distance. As explained in subsection III A, the NLS models 45) and (49) [and Eq. (1)] take into account the effects of Kerr-induced interpulse interaction on radiation dynamics, while the perturbation theory and Eqs. (47) and (51) neglect these effects. Therefore, based on the results shown in Figs. 13,14, we conclude that the effects of Kerr-induced interpulse interaction on radiation dynamics are significant for $\epsilon_{3}=0.02$ and $\beta=10$.

We complete the analysis of the collision-induced radiation dynamics for $\epsilon_{3}=0.02$ and $\beta=10$ by comparing the pulse profiles $\left|\psi_{1}^{(\text {num })}(t, z)\right|,\left|\psi_{1}^{(\text {num }, s)}(t, z)\right|$, and $\left|\psi_{1}^{(\text {num }, p)}(t, z)\right|$ obtained in numerical simulations with the perturbed NLS models (1), (45), and (49), respectively. Figure 15 shows the comparison of these pulse profiles at $z=z_{c}+2, z=z_{c}+5$, and $z=z_{c}+10$. Figure 16 shows magnified versions of the graphs in Fig. 15 for small $\left|\psi_{1}(t, z)\right|$ values. We observe that the pulse profiles obtained with the simplified NLS models (45) and (49) are in good agreement with the result obtained in simulations with the full coupled-NLS model (1), although the agreement is not as good as the one obtained for 

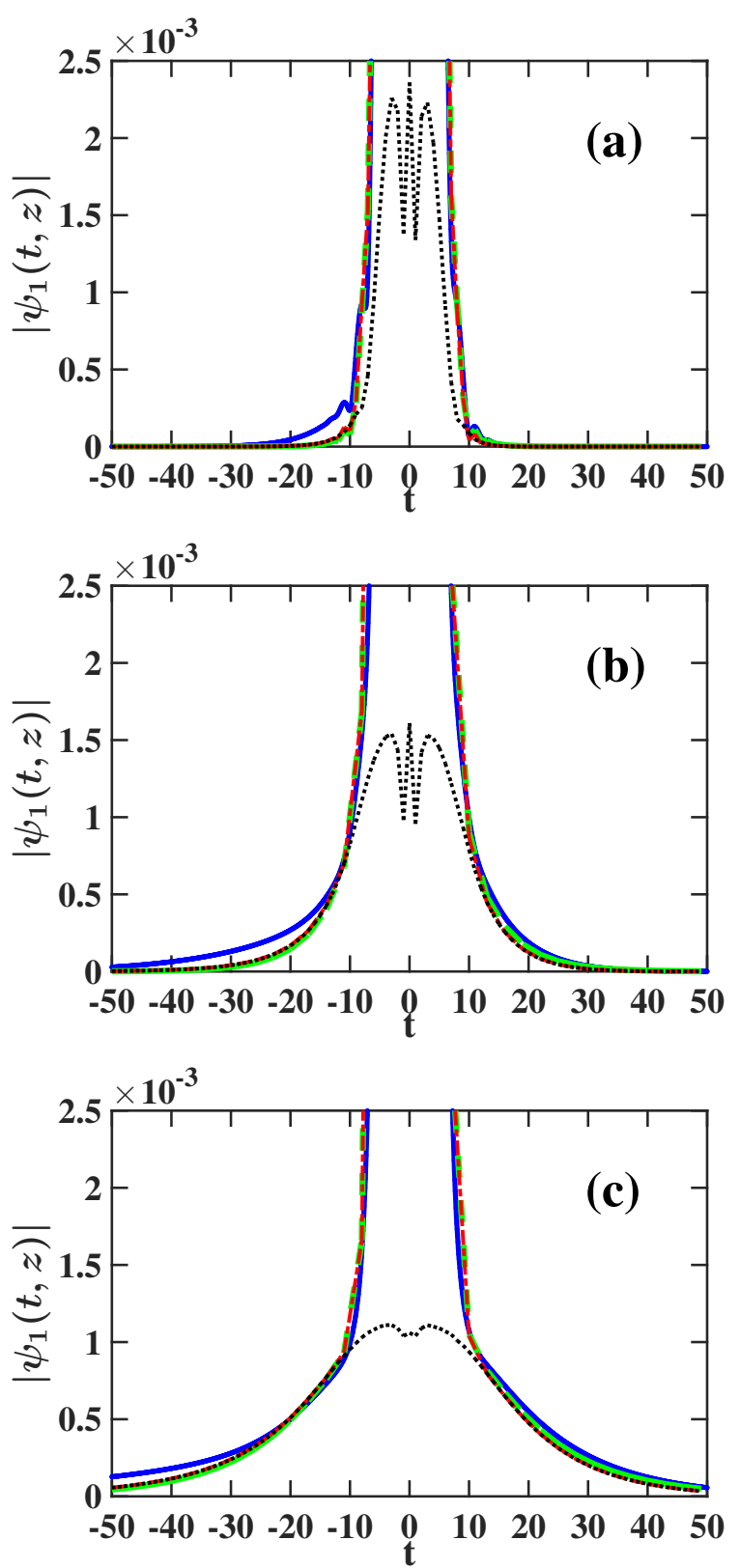

FIG. 11: A comparison between the perturbation theory's predictions and the result of numerical simulation with Eq. (1) for the $t$ dependence of the pulse profile $\left|\psi_{1}(t, z)\right|$. The physical parameter values are $\epsilon_{3}=0.02$ and $\beta=10$ and the distances are $z=z_{c}+2$ in (a), $z=z_{c}+5$ in (b), and $z=z_{c}+10$ in (c). The solid blue curve represents the result obtained by numerical solution of Eq. (1). The dashed green and dashed-dotted red curves correspond to the predictions of the basic and improved versions of the perturbation approach $\left|\psi_{1 b}(t, z)\right|$ and $\left|\psi_{1 c}(t, z)\right|$, respectively. The dotted black curve corresponds to the radiation profile $\left|v_{12 c}(t, z)\right|$ obtained with the improved perturbation procedure. 

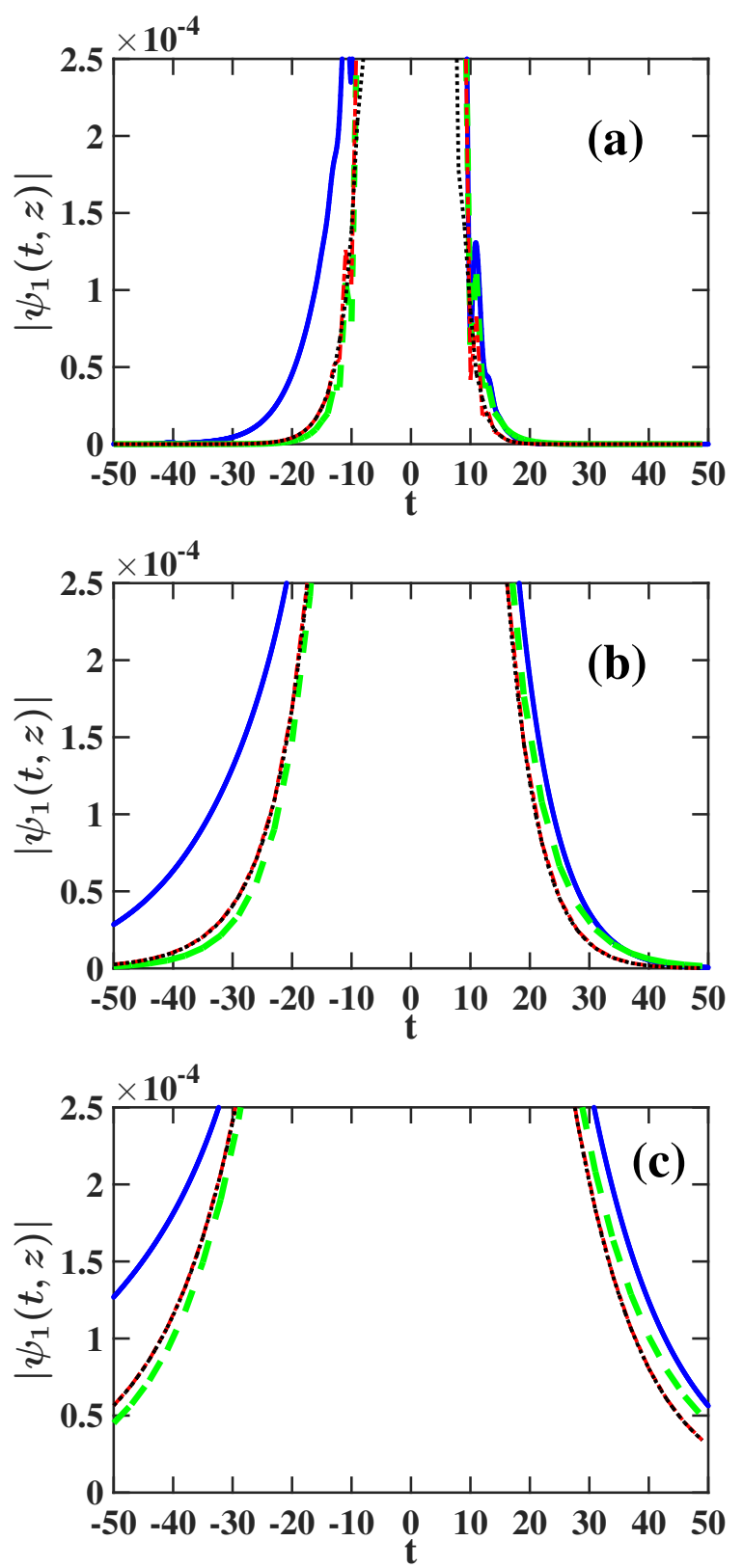

FIG. 12: Magnified versions of the graphs in Fig. 11 for small $\left|\psi_{1}(t, z)\right|$ values. The symbols are the same as in Fig. 11.

$\epsilon_{3}=0.02$ and $\beta=20$ (compare Figs. 15 and 16 with Figs. 9 and 10). In particular, the deviations ||$\psi_{1}^{(\text {num })}(t, z)|-| \psi_{1}^{(\text {num }, s)}(t, z)||$ and ||$\psi_{1}^{(\text {num })}(t, z)|-| \psi_{1}^{(\text {num }, p)}(t, z) \|$ are smaller than $1.36 \times 10^{-3}$ and $1.37 \times 10^{-3}$ for all $t$ values at $z=z_{c}+2$, and are smaller than $3.79 \times 10^{-3}$ and $3.74 \times 10^{-3}$ for all $t$ values at $z=z_{c}+10$. Furthermore, the deviations for $|t|>6$ are smaller than $8.95 \times 10^{-4}$ and $8.96 \times 10^{-4}$ at $z=z_{c}+2$, and are smaller than $3.67 \times 10^{-4}$ and $3.50 \times 10^{-4}$ at $z=z_{c}+10$. Similar to the situation for $\epsilon_{3}=0.02$ and $\beta=20$, the good 

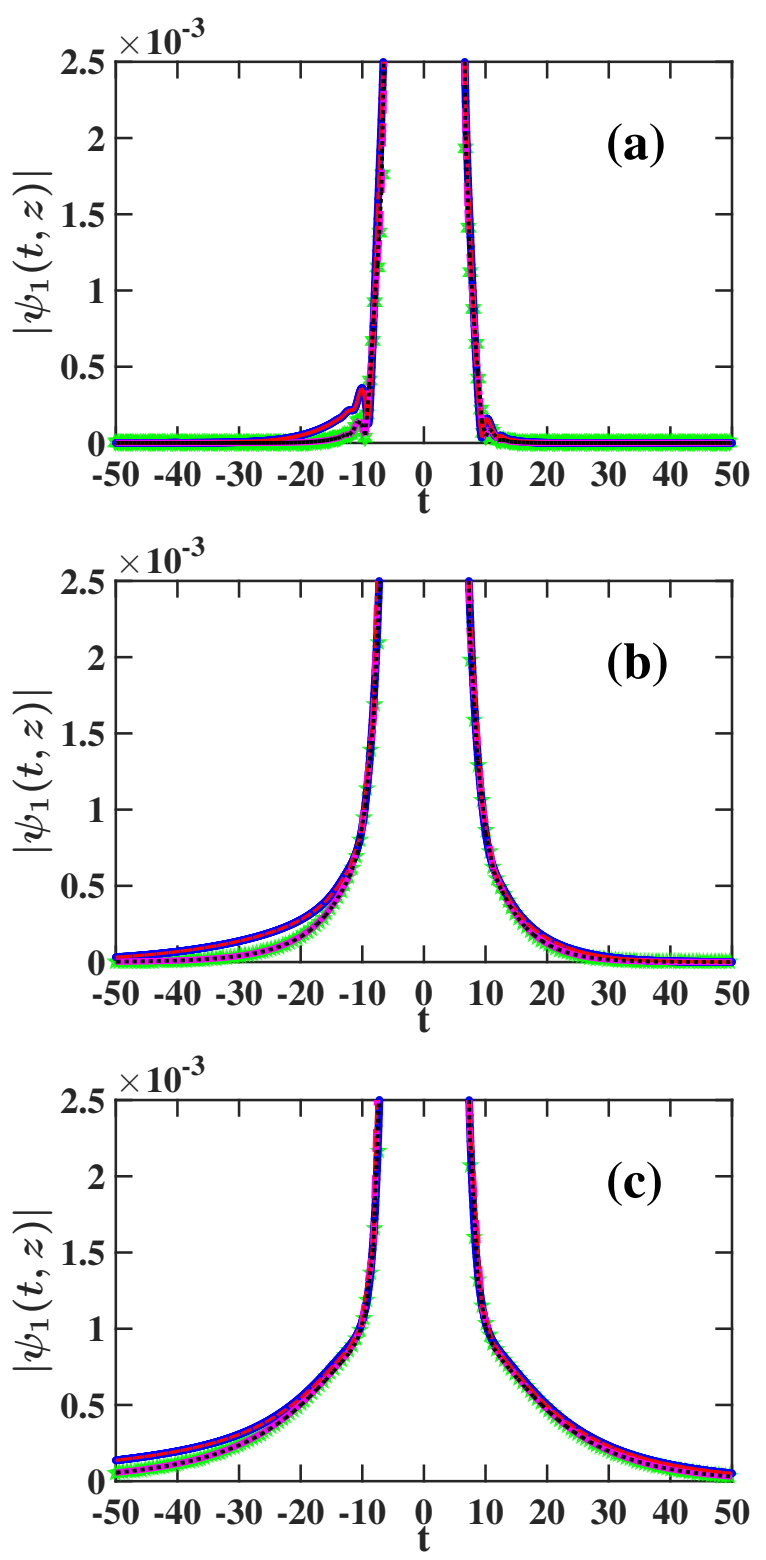

FIG. 13: A comparison between the $t$ dependences of the pulse profile $\left|\psi_{1}(t, z)\right|$ obtained by the improved perturbation theory and the pulse profiles obtained by numerical solution of the four simplified NLS models (45), (47), (49), and (51). The parameter values are $\epsilon_{3}=0.02$ and $\beta=10$ and the distances are $z=z_{c}+2$ in (a), $z=z_{c}+5$ in (b), and $z=z_{c}+10$ in (c). The green stars represent the prediction of the improved perturbation approach. The solid-blue, dashed magenta, dashed-dotted red, and dotted black curves correspond to the pulse profiles obtained by numerical solution of Eqs. 45), 47), 49), and (51). 

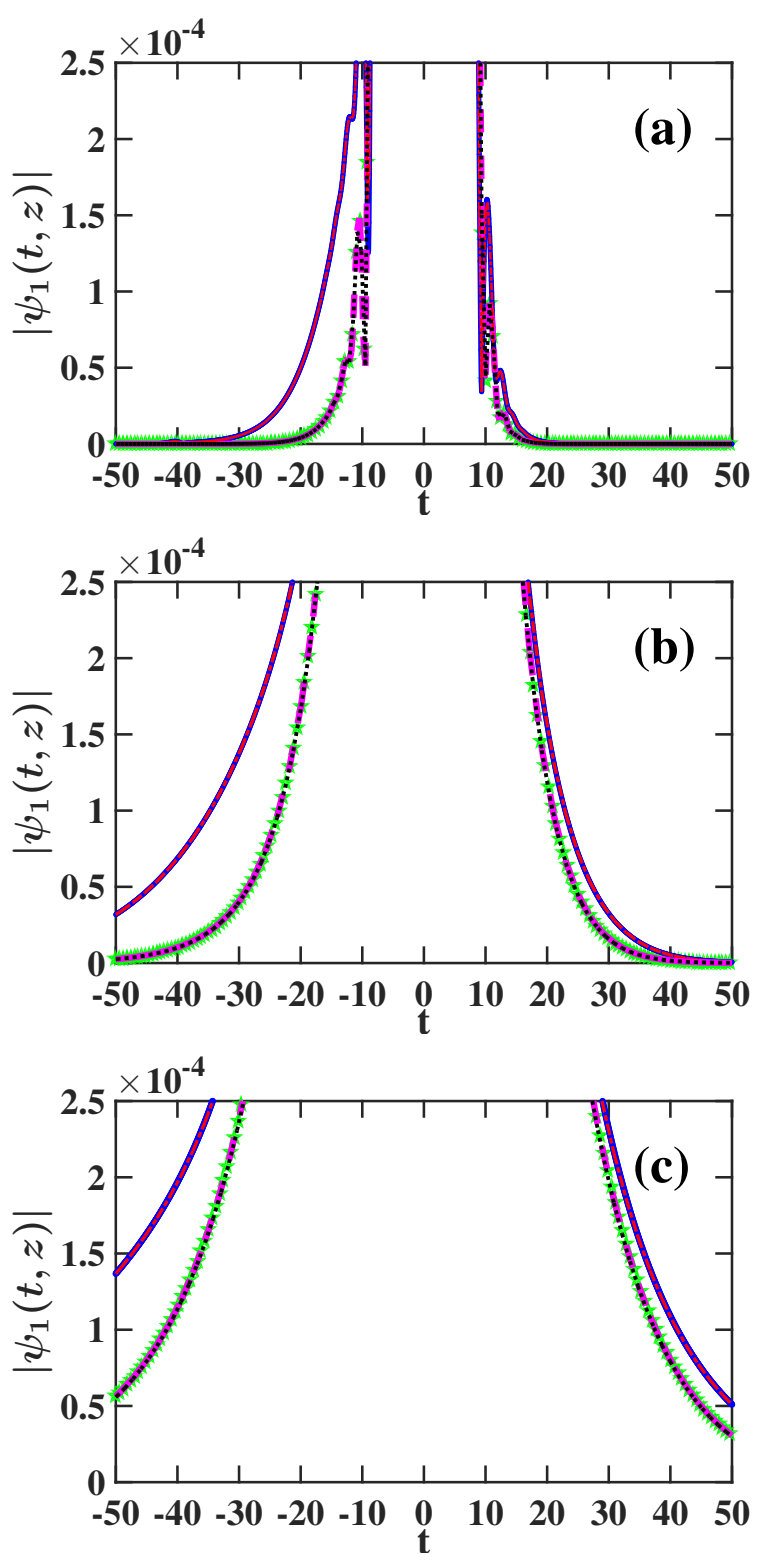

FIG. 14: Magnified versions of the graphs in Fig. 13 for small $\left|\psi_{1}(t, z)\right|$ values. The symbols are the same as in Fig. 13 .

agreement between the pulse profiles obtained with Eqs. (1) and (45) shows that distortion of soliton 2 does not play an important role in the collision-induced radiation dynamics of soliton 1. Furthermore, based on the comparison in Figs. 15 and 16, we conclude that for $\epsilon_{3}=0.02$ and $\beta=10$, we can use the two simplified models 45 and 49 to describe the collision-induced radiation dynamics with good accuracy.

The differences between the perturbation theory predictions for the pulse tails and the pulse tails obtained in the simulations with Eq. (1) can be explained with the help of 

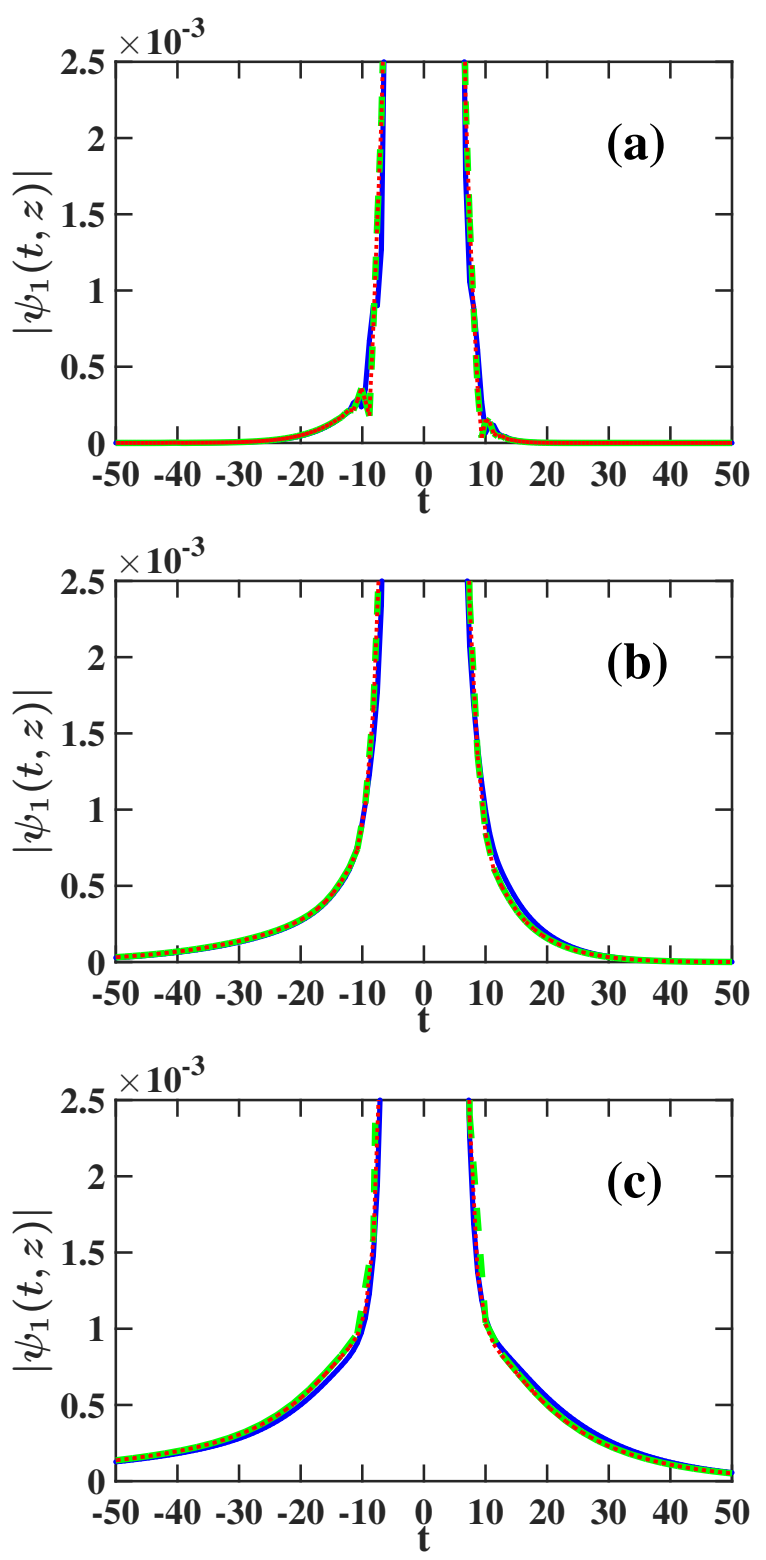

FIG. 15: A comparison between the $t$ dependences of the pulse profile $\left|\psi_{1}(t, z)\right|$ obtained in numerical simulations with the three perturbed NLS models (1), (45), and (49). The physical parameter values are $\epsilon_{3}=0.02$ and $\beta=10$ and the distances are $z=z_{c}+2$ in (a), $z=z_{c}+5$ in (b), and $z=z_{c}+10$ in (c). The solid blue, dashed green, and dotted red curves represent $\left|\psi_{1}(t, z)\right|$ obtained by numerical solution of Eqs. (1), (45), and (49), respectively.

the results shown in Figs. 13 - 16. In particular, in Figs. 13 - 14, we found similar differences between the pulse tails obtained with Eqs. (45) and (49) and the perturbation theory predictions. In contrast, the latter predictions were in good agreement with results 

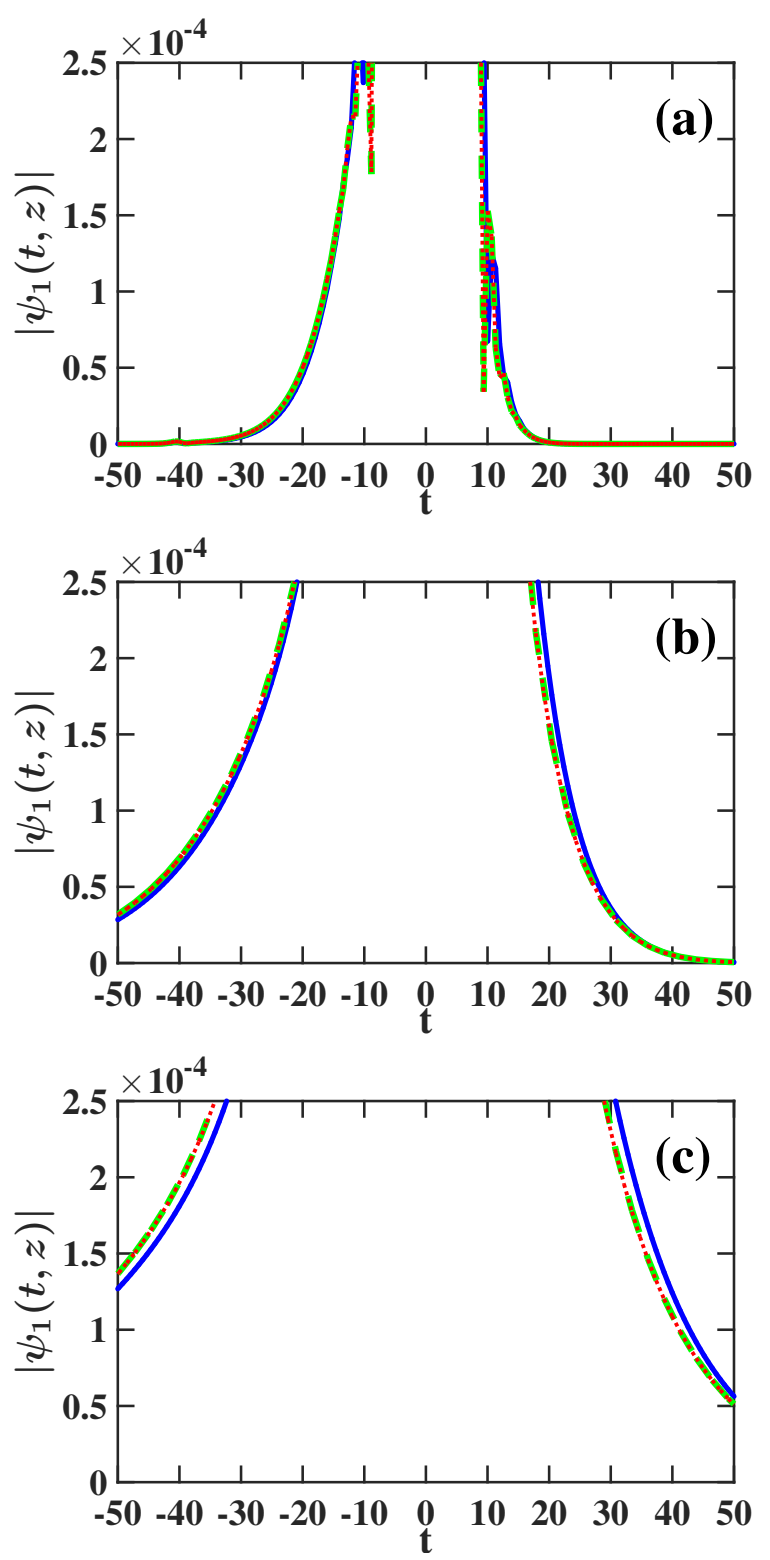

FIG. 16: Magnified versions of the graphs in Fig. 15 for small $\left|\psi_{1}(t, z)\right|$ values. The symbols are the same as in Fig. 15.

of simulations with Eqs. (47) and (51). Furthermore, in Figs. 15 - 16, we found good agreement between the results of the simulations with Eqs. (1), (45), and (49). Recall that the perturbed NLS models (1), (45), and (49) take into account the effects of interpulse interaction due to Kerr nonlinearity on radiation dynamics, while the perturbation theory and the perturbed NLS models (47) and (51) neglect these effects. Therefore, based on the comparisons in Figs. 13 - 16, we conclude that the effects of Kerr-induced interpulse interaction on radiation dynamics are the main cause for the observed differences between 
the pulse tails obtained with the full coupled-NLS model (1) and the pulse tails predicted by the perturbation theory for $\epsilon_{3}=0.02$ and $\beta=10$.

\section{CONCLUSIONS}

We studied the dynamics of emission of radiation in fast collisions between solitons of the NLS equation in the presence of weak cubic loss. We calculated the radiation dynamics and the pulse profile by a perturbation technique with two small parameters: the cubic loss coefficient $\epsilon_{3}$ and the reciprocal of the group velocity difference $1 / \beta$. We then compared the predictions of the perturbation theory with results of numerical simulations with the full propagation equation [the full coupled-NLS model (1)]. The comparison showed very good agreement between the perturbation theory predictions and the simulations with Eq. (1) for large values of $\beta(\beta=20)$. For intermediate values of $\beta(\beta=10)$, we obtained good agreement between the predictions of the perturbation theory and the results of Eq. (1), but the agreement was not as good as the one obtained for $\beta=20$. Therefore, our study provides the first demonstration that the perturbation technique developed in Refs. [17, 23, 27] for studying radiation dynamics in fast soliton collisions in the presence of conservative perturbations can in fact be employed for soliton collisions in the presence of dissipative perturbations.

To gain further insight into the reasons for the differences between the perturbation theory predictions and the results of simulations with Eq. (1), we carried out numerical simulations with four simplified propagation models: two single-NLS models and two simpler coupledNLS models. The first single-NLS model [Eq. (45)] and the first simplified coupled-NLS model [Eq. 49] ] take into account both collision-induced emission of radiation and collisioninduced position shift due to Kerr nonlinearity. The second single-NLS model [Eq. (47)] and the second simplified coupled-NLS model [Eq. (51)] take into account only the latter effect. For large $\beta$ values $(\beta=20)$, the pulse profiles obtained with the four simplified models were in very good agreement with the predictions of the perturbation theory and with the pulse profile obtained with the full coupled-NLS model (1). In contrast, for intermediate values of $\beta(\beta=10)$, only the two simplified models that take into account the effects of Kerr nonlinearity on the collision-induced radiation dynamics [Eqs. (45) and (49)] were in very good agreement with the result of the full coupled-NLS model (1). It follows that the effects 
of Kerr nonlinearity on the collision-induced radiation dynamics are unimportant for $\beta=20$, but are significant for $\beta=10$, i.e., the strength of these effects increases with decreasing value of $\beta$. Furthermore, it follows that the main reason for the observed differences between the perturbation theory calculations and the simulations with Eq. (1) for $\beta=10$ was the additional emission of radiation due to the effects of Kerr nonlinearity on the collision. We also point out that the simulations with the four simplified NLS models also provided a clear demonstration of the significance of the interplay between Kerr nonlinearity and dissipative processes in dynamics of radiation emitted in collisions between NLS solitons.

As explained in sections IID and III A, the simplified NLS models (36), 45), and 47) provide a description of the collision-induced dynamics in terms of a single perturbed NLS equation. In previous studies, we used a generalized form of such perturbed NLS models to describe soliton propagation in multisequence nonlinear optical waveguide systems, where each soliton undergoes many collisions with solitons from all other pulse sequences [16, 34, 35]. The generalized model used in Refs. [16, 34, 35] had the form of a perturbed stochastic NLS equation with a linear gain-loss term and a stochastic (distance-dependent) linear gain-loss coefficient. The linear gain-loss term described the energy exchange of a given soliton in many collisions with solitons from all other pulse sequences. The distancedependent stochastic coefficient of this term described the bit-pattern randomness of the pulse sequences [16, 34, 35]. It was assumed in these studies that the stochastic linear gain-loss term accurately describes collision-induced radiation dynamics. The results of the current paper provide the first strong evidence in favor of the validity of this assumption. More specifically, the good agreement between the pulse profiles obtained in numerical simulations with the single-NLS model (47) and with the full coupled-NLS model (1) shows that the stochastic linear gain-loss term of the NLS models used in Refs. [16, 34, 35] correctly captures the dynamics of the radiation emitted in soliton collisions.

\section{Acknowledgments}

D.C. is grateful to the Mathematics Department of NJCU for providing technological support for the computations. 


\section{Appendix A: The adiabatic perturbation theory for the fundamental NLS soliton}

We provide a summary of the adiabatic perturbation theory for the fundamental NLS soliton, which was developed by Kaup [60, 61, 65]. The theory was used for studying soliton propagation in the presence of perturbations in a variety of nonlinear optical waveguide systems (see, e.g., Refs. [8, 16, 26] and references therein).

We illustrate the approach by considering the following perturbed NLS equation

$$
i \partial_{z} \psi+\partial_{t}^{2} \psi+2|\psi|^{2} \psi=\epsilon h(t, z),
$$

where $0<|\epsilon| \ll 1$. We look for a solution of Eq. (A1) in the form:

$$
\begin{aligned}
\psi(t, z)=\psi_{s}(t, z)+\psi_{\text {rad }}(t, z) & =\eta(z) \frac{\exp [i \chi(t, z)]}{\cosh (x)} \\
& +v(t, z) \exp [i \chi(t, z)],
\end{aligned}
$$

where $x=\eta(z)[t-y(z)], \chi(t, z)=\alpha(z)-\beta(z)[t-y(z)], y(z)=y(0)-2 \int_{0}^{z} d z^{\prime} \beta\left(z^{\prime}\right)$, and $\alpha(z)=\alpha(0)+\int_{0}^{z} d z^{\prime}\left[\eta^{2}\left(z^{\prime}\right)+\beta^{2}\left(z^{\prime}\right)\right]$. The first term on the right hand side of Eq. A2 has the form of a fundamental soliton solution of the NLS equation with slow varying parameters, while the second term, which is of $O(\epsilon)$, is the radiation part. We now substitute Eq. A2 into Eq. (A1) and keep terms up to $O(\epsilon)$. The equation obtained by this substitution and the complex conjugate of this equation can be written in the following vector form:

$$
\begin{aligned}
& \frac{i}{\cosh (x)}\left(\begin{array}{c}
1 \\
-1
\end{array}\right) \eta\left(\frac{d \alpha}{d z}+\beta \frac{d y}{d z}-\eta^{2}+\beta^{2}\right) \\
& +\frac{\tanh (x)}{\cosh (x)}\left(\begin{array}{c}
1 \\
1
\end{array}\right) \eta^{2}\left(\frac{d y}{d z}+2 \beta\right)-\frac{i x}{\cosh (x)}\left(\begin{array}{c}
1 \\
-1
\end{array}\right) \frac{d \beta}{d z} \\
& -\frac{[x \tanh (x)-1]}{\cosh (x)}\left(\begin{array}{c}
1 \\
1
\end{array}\right) \frac{d \eta}{d z}+\partial_{z}\left(\begin{array}{c}
v \\
v^{*}
\end{array}\right)-i \eta^{2} \mathcal{L}\left(\begin{array}{c}
v \\
v^{*}
\end{array}\right) \\
& -2 \beta \partial_{t}\left(\begin{array}{c}
v \\
v^{*}
\end{array}\right)=-i \epsilon\left(\begin{array}{c}
h(t, z) e^{-i \chi} \\
-h^{*}(t, z) e^{i \chi}
\end{array}\right) .
\end{aligned}
$$

The linear operator $\mathcal{L}$ in Eq. A3 is defined by:

$$
\mathcal{L}=\left(\partial_{x}^{2}-1\right) \boldsymbol{\sigma}_{\mathbf{3}}+\frac{2}{\cosh ^{2}(x)}\left(2 \boldsymbol{\sigma}_{\mathbf{3}}+i \boldsymbol{\sigma}_{\mathbf{2}}\right),
$$

where $\boldsymbol{\sigma}_{\boldsymbol{j}}$ with $1 \leq j \leq 3$ are the Pauli spin matrices.

The complete set of orthogonal eigenfunctions of $\mathcal{L}$ was found in Refs. [60, 61, 65]. It includes four localized eigenfunctions, which appear in the first four terms on the left hand 
side of Eq. (A3):

$$
\begin{aligned}
& f_{0}(x)=\frac{1}{\cosh (x)}\left(\begin{array}{c}
1 \\
-1
\end{array}\right), f_{1}(x)=\frac{\tanh (x)}{\cosh (x)}\left(\begin{array}{l}
1 \\
1
\end{array}\right) \\
& f_{2}(x)=\frac{x}{\cosh (x)}\left(\begin{array}{c}
1 \\
-1
\end{array}\right), f_{3}(x)=\frac{x \tanh (x)-1}{\cosh (x)}\left(\begin{array}{l}
1 \\
1
\end{array}\right)
\end{aligned}
$$

The eigenfunctions $f_{0}(x)$ and $f_{1}(x)$ have a zero eigenvalue, while $f_{2}(x)$ and $f_{3}(x)$ satisfy $\mathcal{L} f_{2}=-2 f_{1}$ and $\mathcal{L} f_{3}=-2 f_{0}$ [60, 61, 65]. The left localized eigenfunctions of $\mathcal{L}$, which are given by $f_{m}^{T} \boldsymbol{\sigma}_{\mathbf{3}}$ for $0 \leq m \leq 3$, satisfy the following relations [60, 61, 65]:

$$
\begin{gathered}
\int_{-\infty}^{+\infty} d x f_{2}^{T}(x) \boldsymbol{\sigma}_{\mathbf{3}} f_{1}(x)=2, \\
\int_{-\infty}^{+\infty} d x f_{0}^{T}(x) \boldsymbol{\sigma}_{\mathbf{3}} f_{3}(x)=-2 .
\end{gathered}
$$

We obtain the dynamic equations for the four soliton parameters by projecting both sides of Eq. A3 on the four left localized eigenfunctions of $\mathcal{L}$.

The set of eigenfunctions of $\mathcal{L}$ also contains an infinite set of nonlocalized eigenfunctions $f_{s}(x)$ and $\bar{f}_{s}(x)$ with eigenvalues $s^{2}+1$ and $-\left(s^{2}+1\right)$, respectively, where $-\infty<s<\infty$, and $\bar{f}_{s}(x) \equiv \sigma_{1} f_{s}^{*}(x)$. The eigenfunctions $f_{s}(x)$ and $\bar{f}_{s}(x)$ are given by [60, 61, 65]:

$$
\begin{aligned}
& f_{s}(x)=\exp (i s x)\left[1-\frac{2 i s \exp (-x)}{(s+i)^{2} \cosh (x)}\right]\left(\begin{array}{l}
0 \\
1
\end{array}\right) \\
& +\frac{\exp (i s x)}{(s+i)^{2} \cosh ^{2}(x)}\left(\begin{array}{l}
1 \\
1
\end{array}\right)
\end{aligned}
$$

and

$$
\begin{aligned}
& \bar{f}_{s}(x)=\exp (-i s x)\left[1+\frac{2 i s \exp (-x)}{(s-i)^{2} \cosh (x)}\right]\left(\begin{array}{l}
1 \\
0
\end{array}\right) \\
& +\frac{\exp (-i s x)}{(s-i)^{2} \cosh ^{2}(x)}\left(\begin{array}{l}
1 \\
1
\end{array}\right) .
\end{aligned}
$$

The corresponding left eigenfunctions of $\mathcal{L}$ are given by $f_{s}^{+}(x) \boldsymbol{\sigma}_{3}$ and $\bar{f}_{s}^{+}(x) \boldsymbol{\sigma}_{3}$. These eigenfunctions satisfy the relations [60, 61, 65]

$$
\begin{aligned}
& \int_{-\infty}^{+\infty} d x f_{s}^{+}(x) \sigma_{3} f_{s^{\prime}}(x)=-2 \pi \delta\left(s-s^{\prime}\right) \\
& \int_{-\infty}^{+\infty} d x \bar{f}_{s}^{+}(x) \sigma_{3} \bar{f}_{s^{\prime}}(x)=2 \pi \delta\left(s-s^{\prime}\right)
\end{aligned}
$$


To obtain the dynamics of the emitted radiation, we expand $v(t, z)$ and $\epsilon h(t, z)$ in the nonlocalized eigenfunctions of $\mathcal{L}$ :

$$
\left(\begin{array}{c}
v(t, z) \\
v^{*}(t, z)
\end{array}\right)=\int_{-\infty}^{+\infty} \frac{d s}{2 \pi}\left[a_{s}(z) f_{s}(x)+a_{s}^{*}(z) \bar{f}_{s}(x)\right],
$$

and

$$
-i \epsilon\left(\begin{array}{c}
h(t, z) e^{-i \chi} \\
-h^{*}(t, z) e^{i \chi}
\end{array}\right)=\int_{-\infty}^{+\infty} \frac{d s}{2 \pi}\left[b_{s}(z) f_{s}(x)+b_{s}^{*}(z) \bar{f}_{s}(x)\right] .
$$

We then substitute the expansions $\mathrm{A} 12$ and $\mathrm{A} 13$ into Eq. A3 and project both sides of the resultant equation on the left nonlocalized eigenfunctions of $\mathcal{L}, f_{s}^{+}(x) \boldsymbol{\sigma}_{3}$. This calculation yields the evolution equation for the expansion coefficients $a_{s}(z)$ :

$$
\frac{d a_{s}(z)}{d z}-i \eta^{2}\left(s^{2}+1\right) a_{s}(z)=b_{s}(z)
$$

\section{Appendix B: The form of the four simplified NLS models of section III}

In this appendix, we explain the form of the four simplified NLS models (45), (47), (49), and (51) that are used in section III to gain further insight into the collision-induced radiation dynamics. More specifically, the simplified models are used for the following two reasons. (a) To enable accurate identification of the most important corrections to the perturbative clacluations of radiation dynamics in section II. (b) To help determine the significance of radiation-induced distortion of soliton 2 for radiation dynamics of soliton 1. It turns out (from numerical simulations with the simplified models) that the most important correction to the perturbative calculations of section II] is due to the effects of interpulse interaction induced by Kerr nonlinearity. Therefore, the form of the simplified NLS models that we use must help identify the role of interpulse interaction due to Kerr nonlinearity in radiation dynamics. In addition, in order to enable accurate comparison with the results of numerical simulations with the full coupled-NLS model (1) and with the perturbation theory predictions, the simplified NLS models must take into account the main collision-induced position shifts.

We achieve the two goals specified in the beginning of the preceding paragraph by using two perturbed single-NLS models and two perturbed coupled-NLS models as the simplified propagation models. The two perturbed single-NLS models [Eqs. (45) and (47)] neglect the effects of radiation-induced distortion of soliton 2 on radiation dynamics of soliton 1 . 
Therefore, in these models, $\psi_{2}(t, z)$ is replaced by the corresponding fundamental soliton solution of the unperturbed NLS equation $\psi_{s 2}(t, z)$. As a result, the term $-2 i \epsilon_{3}\left|\psi_{2}\right|^{2} \psi_{1}$ in Eq. (1) turns into the term $-\left(2 i \epsilon_{3} \eta_{2}^{2} \psi_{1}\right) /\left[\cosh ^{2}\left(x_{2}\right)\right]$ in Eqs. (45) and (47). The singleNLS model (45) takes into account the effects of Kerr nonlinearity on both collision-induced radiation dynamics and collision-induced position shifts. This is achieved by turning the term $4\left|\psi_{2}\right|^{2} \psi_{1}$ on the left hand side of Eq. (1), which is associated with the latter effects, into the term $-\left(4 \eta_{2}^{2} \psi_{1}\right) /\left[\cosh ^{2}\left(x_{2}\right)\right]$ on the right hand side of Eq. (45). Next, we incorporate the position shift arising from the collision-induced frequency shift experienced by soliton 1 into the model. For this purpose, we first note that the latter frequency shift is given by [29]: $\Delta \beta_{13}^{(1)}=-\left(20 \epsilon_{3} \eta_{1}^{2} \eta_{2}\right) /(3|\beta| \beta)$. Therefore, by the adiabatic perturbation theory for the fundamental NLS soliton, the associated position shift of soliton $1, y_{1}^{(C)}(z)$, satisfies the equations $d y_{1}^{(C)} / d z=0$ for $z<z_{c}$, and $d y_{1}^{(C)} / d z=-2 \Delta \beta_{13}^{(1)}=\left(40 \epsilon_{3} \eta_{1}^{2} \eta_{2}\right) /(3|\beta| \beta)$ for $z \geq z_{c}$. Consequently, $y_{1}^{(C)}(z)$ is given by:

$$
y_{1}^{(C)}(z)= \begin{cases}0 & \text { for } z<z_{c}, \\ \frac{40 \epsilon_{3} \eta_{1}^{2} \eta_{2}}{3|\beta| \beta}\left(z-z_{c}\right) & \text { for } z \geq z_{c},\end{cases}
$$

a result that we use in Eqs. (21) and (32) for $\psi_{1 b}(t, z)$ and $\psi_{1 c}(t, z)$. On the other hand, it is known that a position shift, which is not accompanied by a change in the soliton's shape, amplitude, and frequency, is described by a term of the form $-i C_{1}(z) \partial_{t} \psi_{1}$ (see, e.g., Ref. [35]). The position shift induced by the latter perturbation term satisfies $d y_{1}^{(C)} / d z=C_{1}(z)$. Equating the right hand sides of the two equations for $d y_{1}^{(C)} / d z$, we arrive at:

$$
C_{1}(z)= \begin{cases}0 & \text { for } z<z_{c} \\ \frac{40 \epsilon_{3} \eta_{1}^{2} \eta_{2}}{3|\beta| \beta} & \text { for } z \geq z_{c}\end{cases}
$$

which is Eq. (46). Combining all the aforementioned approximations for the full coupledNLS propagation model, we find that the first simplified single-NLS model is given by Eq. (45), that is:

$$
i \partial_{z} \psi_{1}+\partial_{t}^{2} \psi_{1}+2\left|\psi_{1}\right|^{2} \psi_{1}=-\frac{2 i \epsilon_{3} \eta_{2}^{2}}{\cosh ^{2}\left(x_{2}\right)} \psi_{1}-\frac{4 \eta_{2}^{2}}{\cosh ^{2}\left(x_{2}\right)} \psi_{1}-i C_{1}(z) \partial_{t} \psi_{1} .
$$

The second simplified single-NLS model [Eq. (47)] takes into account the effects of cubic loss on collision-induced radiation dynamics and the position shift arising from the collision-induced frequency shift in exactly the same manner as in the first single-NLS model 
[Eq. 445]]. Thus, these effects are described by the terms $-\left(2 i \epsilon_{3} \eta_{2}^{2} \psi_{1}\right) /\left[\cosh ^{2}\left(x_{2}\right)\right]$ and $-i C_{1}(z) \partial_{t} \psi_{1}$ on the right hand side of Eq. (47), where $C_{1}(z)$ is given by Eq. (46). In addition, the second single-NLS model takes into account the effects of Kerr nonlinearity on the collision-induced position shift, but neglects the effects of Kerr nonlinearity on the collision-induced radiation dynamics. Therefore, in this case, the term $4\left|\psi_{2}\right|{ }^{2} \psi_{1}$ on the left hand side of Eq. (1) is replaced by a term of the form $-i C_{2}(z) \partial_{t} \psi_{1}$ on the right hand side of Eq. (47). Combining all the approximations for the full coupled-NLS propagation model, we find that the second simplified single-NLS model is given by:

$$
i \partial_{z} \psi_{1}+\partial_{t}^{2} \psi_{1}+2\left|\psi_{1}\right|^{2} \psi_{1}=-\frac{2 i \epsilon_{3} \eta_{2}^{2}}{\cosh ^{2}\left(x_{2}\right)} \psi_{1}-i C_{2}(z) \partial_{t} \psi_{1}-i C_{1}(z) \partial_{t} \psi_{1},
$$

which is Eq. (47). The function $C_{2}(z)$ is calculated in a similar manner to $C_{1}(z)$. For this purpose, we note that the collision-induced position shift experienced by soliton 1 due to the effects of Kerr nonlinearity is given by Eq. (7). For simplicity, we assume that the contribution of the collision-induced position shift to the total position shift of soliton 1 , $y_{1}^{(K)}(z)$, changes linearly from 0 to $4 \eta_{2} /(\beta|\beta|)$ in the small interval $\left[z_{c}-1 /(2|\beta|), z_{c}+1 /(2|\beta|)\right]$ about the collision point $z_{c}$. It follows that $y_{1}^{(K)}(z)$ is given by: $y_{1}^{(K)}(z)=0$ for $z<$ $z_{c}-1 /(2|\beta|), y_{1}^{(K)}(z)=4 \eta_{2} z /|\beta|-\left\{2 \eta_{2}\left[y_{2}(0)-1\right]\right\} /(|\beta| \beta)$ for $z_{c}-1 /(2|\beta|) \leq z \leq z_{c}+1 /(2|\beta|)$, and $y_{1}^{(K)}(z)=4 \eta_{2} /(\beta|\beta|)$ for $z>z_{c}+1 /(2|\beta|)$. As a result, $d y_{1}^{(K)} / d z=0$ for $z<z_{c}-1 /(2|\beta|)$ and $z>z_{c}+1 /(2|\beta|)$, and $d y_{1}^{(K)} / d z=4 \eta_{2} /|\beta|$ for $z_{c}-1 /(2|\beta|) \leq z \leq z_{c}+1 /(2|\beta|)$. On the other hand, $d y_{1}^{(K)} / d z=C_{2}(z)$. Equating the right hand sides of the eqations for $d y_{1}^{(K)} / d z$, we obtain

$$
C_{2}(z)= \begin{cases}0 & \text { for } z<z_{c}-\frac{1}{2|\beta|} \\ \frac{4 \eta_{2}}{|\beta|} & \text { for } z_{c}-\frac{1}{2|\beta|} \leq z \leq z_{c}+\frac{1}{2|\beta|} \\ 0 & \text { for } z>z_{c}+\frac{1}{2|\beta|}\end{cases}
$$

which is Eq. 48).

The two simplified coupled-NLS models [Eqs. (49) and (51)] take into account the effects of radiation-induced distortion of soliton 2 on radiation dynamics of soliton 1 in the leading order. That is, these effects are taken into account for radiation dynamics induced by cubic loss, but are neglected for radiation dynamics induced by Kerr nonlinearity. Consequently, the cubic loss terms of the full coupled-NLS model (1), $-2 i \epsilon_{3}\left|\psi_{2}\right|^{2} \psi_{1}$ and $-2 i \epsilon_{3}\left|\psi_{1}\right|^{2} \psi_{2}$, appear unchanged in Eqs. (49) and (51). The first simplified coupled-NLS model [Eq. 
(49)] takes into account the effects of Kerr nonlinearity on both collision-induced radiation dynamics and collision-induced position shifts of each soliton. Therefore, the terms $4\left|\psi_{2}\right|^{2} \psi_{1}$ and $4\left|\psi_{1}\right|^{2} \psi_{2}$ on the left hand side of Eq. (1), turn into the terms $-\left(4 \eta_{2}^{2} \psi_{1}\right) /\left[\cosh ^{2}\left(x_{2}\right)\right]$ and $-\left(4 \eta_{1}^{2} \psi_{2}\right) /\left[\cosh ^{2}\left(x_{1}\right)\right]$ on the right hand side of Eq. (49). The position shifts arising from the collision-induced frequency shifts are taken into account in the same manner as in the simplified single-NLS models (45) and (47). That is, we include terms of the form $-i C_{1}(z) \partial_{t} \psi_{1}$ and $-i C_{3}(z) \partial_{t} \psi_{2}$ on the right hand side of Eq. 49$)$, where $C_{1}(z)$ and $C_{3}(z)$ are given by Eqs. (46) and (50), respectively [66]. Taking into account all the approximations for the full coupled-NLS model, we find that the first simplified coupled-NLS model is given by:

$$
\begin{aligned}
& i \partial_{z} \psi_{1}+\partial_{t}^{2} \psi_{1}+2\left|\psi_{1}\right|^{2} \psi_{1}=-2 i \epsilon_{3}\left|\psi_{2}\right|^{2} \psi_{1}-\frac{4 \eta_{2}^{2}}{\cosh ^{2}\left(x_{2}\right)} \psi_{1}-i C_{1}(z) \partial_{t} \psi_{1} \\
& i \partial_{z} \psi_{2}+\partial_{t}^{2} \psi_{2}+2\left|\psi_{2}\right|^{2} \psi_{2}=-2 i \epsilon_{3}\left|\psi_{1}\right|^{2} \psi_{2}-\frac{4 \eta_{1}^{2}}{\cosh ^{2}\left(x_{1}\right)} \psi_{2}-i C_{3}(z) \partial_{t} \psi_{2}
\end{aligned}
$$

which is Eq. (49).

The only difference between the second and first simplified coupled-NLS models is in the description of the effects of Kerr nonlinearity on the collision-induced dynamics. More specifically, the second simplified coupled-NLS model [Eq. (51)] takes into account only the effects of Kerr nonlinearity on the collision-induced position shifts, while the effects of Kerr nonlinearity on the collision-induced radiation dynamics are neglected. Therefore, the terms $4\left|\psi_{2}\right|^{2} \psi_{1}$ and $4\left|\psi_{1}\right|^{2} \psi_{2}$ on the left hand side of Eq. (1) are replaced by the terms $-i C_{2}(z) \partial_{t} \psi_{1}$ and $-i C_{4}(z) \partial_{t} \psi_{2}$ on the right hand side of Eq. (51), where $C_{2}(z)$ and $C_{4}(z)$ are given by Eqs. (48) and (52), respectively [67]. Combining all the approximations for Eq. (1), we find that the second simplified coupled-NLS model is given by:

$$
\begin{aligned}
& i \partial_{z} \psi_{1}+\partial_{t}^{2} \psi_{1}+2\left|\psi_{1}\right|^{2} \psi_{1}=-2 i \epsilon_{3}\left|\psi_{2}\right|^{2} \psi_{1}-i C_{2}(z) \partial_{t} \psi_{1}-i C_{1}(z) \partial_{t} \psi_{1}, \\
& i \partial_{z} \psi_{2}+\partial_{t}^{2} \psi_{2}+2\left|\psi_{2}\right|^{2} \psi_{2}=-2 i \epsilon_{3}\left|\psi_{1}\right|^{2} \psi_{2}-i C_{4}(z) \partial_{t} \psi_{2}-i C_{3}(z) \partial_{t} \psi_{2}
\end{aligned}
$$

which is Eq. (51).

[1] S. Novikov, S.V. Manakov, L.P. Pitaevskii, and V.E. Zakharov, Theory of Solitons: The Inverse Scattering Method (Plenum, New York, 1984). 
[2] A.C. Newell, Solitons in Mathematics and Physics (SIAM, Philadelphia, 1985).

[3] N. Asano, T. Taniuti, and N. Yajima, J. Math. Phys. 10, 2020 (1969)

[4] W. Horton and Y.H. Ichikawa, Chaos and Structure in Nonlinear Plasmas (World Scientific, Singapore, 1996).

[5] F. Dalfovo, S. Giorgini, L.P. Pitaevskii, and S. Stringari, Rev. Mod. Phys. 71, 463 (1999).

[6] R. Carretero-González, D.J. Frantzeskakis, and P.G. Kevrekidis, Nonlinearity 21, R139 (2008).

[7] G.P. Agrawal, Nonlinear Fiber Optics (Academic, San Diego, CA, 2001).

[8] A. Hasegawa and Y. Kodama, Solitons in Optical Communications (Clarendon, Oxford, 1995).

[9] E. Iannone, F. Matera, A. Mecozzi, and M. Settembre, Nonlinear Optical Communication Networks (Wiley, New York, 1998).

[10] L.F. Mollenauer and J.P. Gordon, Solitons in Optical Fibers: Fundamentals and Applications (Academic, San Diego, CA, 2006).

[11] G.P. Agrawal, Applications of Nonlinear Fiber Optics (Academic, San Diego, CA, 2001).

[12] F. Forghieri, R.W. Tkach, and A.R. Chraplyvy, in Optical Fiber Telecommunications III, I.P. Kaminow and T.L. Koch, eds., (Academic, San Diego, CA, 1997), Chapter 8.

[13] R.-J. Essiambre, G. Kramer, P.J. Winzer, G.J. Foschini, and B. Goebel, J. Lightwave Technol. 28, $662(2010)$.

[14] L.F. Mollenauer and P.V. Mamyshev, IEEE J. Quantum Electron. 34, 2089 (1998).

[15] A. Peleg, Q.M. Nguyen, and T.T. Huynh, Eur. Phys. J. D 71, 315 (2017).

[16] Y. Chung and A. Peleg, Nonlinearity 18, 1555 (2005).

[17] A. Peleg, M. Chertkov, and I. Gabitov, Phys. Rev. E 68, 026605 (2003).

[18] Y.S. Kivshar and B.A. Malomed, Rev. Mod. Phys. 61, 763 (1989).

[19] Y.S. Kivshar and B.A. Malomed, J. Phys. A 19, L967 (1986).

[20] B.A. Malomed, Phys. Rev. E 43, 3114 (1991).

[21] B.A. Malomed, Phys. Rev. A 44, 1412 (1991).

[22] S. Kumar, Opt. Lett. 23, 1450 (1998).

[23] J. Soneson and A. Peleg, Physica D 195, 123 (2004).

[24] D. Chakraborty, A. Peleg, and Q.M. Nguyen, Opt. Commun. 371, 252 (2016).

[25] A. Peleg, Q.M. Nguyen, and T.P. Tran, Opt. Commun. 380, 41 (2016).

[26] M. Chertkov, Y. Chung, A. Dyachenko, I. Gabitov, I. Kolokolov, and V. Lebedev, Phys. Rev. E 67, 036615 (2003). 
[27] A. Peleg, M. Chertkov, and I. Gabitov, J. Opt. Soc. Am. B 21, 18 (2004).

[28] A. Peleg and Y. Chung, J. Phys. A 36, 10039 (2003).

[29] A. Peleg, Q.M. Nguyen, and Y. Chung, Phys. Rev. A 82, 053830 (2010).

[30] A. Peleg and Y. Chung, Phys. Rev. A 85, 063828 (2012).

[31] A. Peleg, Opt. Lett. 29, 1980 (2004).

[32] Q.M. Nguyen and A. Peleg, J. Opt. Soc. Am. B 27, 1985 (2010).

[33] This assumption is justified in the penultimate paragraph of the introduction.

[34] Y. Chung and A. Peleg, Phys. Rev. A 77, 063835 (2008).

[35] A. Peleg and Y. Chung, Opt. Commun. 285, 1429 (2012).

[36] R.W. Boyd, Nonlinear Optics (Academic, San Diego, CA, 2008).

[37] Q. Lin, O.J. Painter, and G.P. Agrawal, Opt. Express 15, 16604 (2007).

[38] R. Dekker, N. Usechak, M. Först, and A. Driessen, J. Phys. D 40, R249 (2007).

[39] M. Borghi, C. Castellan, S. Signorini, A. Trenti, and L. Pavesi, J. Opt. 19, 093002 (2017).

[40] V. Mizrahi, K.W. DeLong, G.I. Stegeman, M.A. Saifi, and M.J. Andrejco, Opt. Lett. 14, 1140 (1989).

[41] Y. Silberberg, Opt. Lett. 15, 1005 (1990).

[42] A.B. Aceves and J.V. Moloney, Opt. Lett. 17, 1488 (1992).

[43] V.V. Afanasjev, J.S. Aitchison. and Y.S. Kivshar, Opt. Commun. 116, 331 (1995).

[44] E.N. Tsoy, C.M. de Sterke, and F.Kh. Abdullaev, J. Opt. Soc. Am. B 18, 1144 (2001).

[45] O. Katz, Y. Lahini, and Y. Silberberg, Opt. Lett. 33, 2830 (2008).

[46] A. Peleg, Y. Chung, T. Dohnal, and Q.M. Nguyen, Phys. Rev. E 80, 026602 (2009).

[47] Y. Okawachi, O. Kuzucu, M.A. Foster, R. Salem, A.C. Turner-Foster, A. Biberman, N. Ophir, K. Bergman, M. Lipson, and A.L. Gaeta, IEEE Photon. Technol. Lett. 24, 185 (2012).

[48] M.A. Foster, A.C. Turner, M. Lipson, and A.L. Gaeta, Opt. Express 16, 1300 (2008).

[49] R. Soref, IEEE J. Sel. Top. Quantum Electron. 12, 1678 (2006).

[50] R.A. Negres, J.M. Hales, A. Kobyakov, D.J. Hagan, and E.W. Van Stryland, IEEE J. Quantum Electron. 38, 1205 (2002).

[51] C.M. Cirloganu, L.A. Padilha, D.A. Fishman, S.Webster, D.J. Hagan, and E.W. Van Stryland, Opt. Express 19, 22951 (2011).

[52] C. Rauscher and R. Laenen, J. Appl. Phys. 81, 2818 (1997).

[53] M.N. Islam (Ed.), Raman Amplifiers for Telecommunications 1: Physical Principles (Springer, 
New York, 2004).

[54] C. Headley and G.P. Agrawal (Eds.), Raman Amplification in Fiber Optical Communication Systems (Elsevier, San Diego, CA, 2005).

[55] R. Claps, D. Dimitropoulos, V. Raghunathan, Y. Han, and B. Jalali, Opt. Express 11, 1731 (2003).

[56] Q. Xu, V.R. Almeida, and M. Lipson, Opt. Express 12, 4437 (2004).

[57] R. Jones, H. Rong, A. Liu, A. Fang, M. Paniccia, D. Hak, and O. Cohen, Opt. Express 13, $519(2005)$

[58] The dimensionless distance $z$ in Eq. (1) is $z=X /\left(2 L_{D}\right)$, where $X$ is the dimensional distance, $L_{D}=\tau_{0}^{2} /\left|\tilde{\beta}_{2}\right|$ is the dispersion length, $\tau_{0}$ is the soliton width, and $\tilde{\beta}_{2}$ is the second-order dispersion coefficient. The dimensionless time is $t=\tau / \tau_{0}$, where $\tau$ is time. $\psi_{j}=\left(\gamma \tau_{0}^{2} /\left|\tilde{\beta}_{2}\right|\right)^{1 / 2} E_{j}$, where $E_{j}$ is the electric field of the $j$ th pulse, $j=1,2$, and $\gamma$ is the Kerr nonlinearity coefficient. The coefficient $\epsilon_{3}$ is related to the dimensional cubic loss coefficient $\rho_{3}$ by $\epsilon_{3}=2 \rho_{3} / \gamma$.

[59] M. Nakazawa, IEEE J. Sel. Top. Quantum Electron. 6, 1332 (2000).

[60] D.J. Kaup, Phys. Rev. A 42, 5689 (1990).

[61] D.J. Kaup, Phys. Rev. A 44, 4582 (1991).

[62] J. Yang, Nonlinear Waves in Integrable and Nonintegrable Systems (SIAM, Philadelphia, 2010).

[63] The interval $-10.77 \leq t \leq 21.44$ consists of several subintervals on which one of the two approaches is more accurate than the other.

[64] The interval $-6.28 \leq t \leq 6.54$ consists of several subintervals on which one of the two approaches is more accurate than the other.

[65] D.J. Kaup, J. Math. Anal. Appl. 54, 849 (1976).

[66] Note that the collision-induced frequency shift experienced by soliton 2 is [29]: $\Delta \beta_{23}^{(1)}=$ $\left(20 \epsilon_{3} \eta_{1} \eta_{2}^{2}\right) /(3|\beta| \beta)$. Therefore, Eq. (50) for $C_{3}(z)$ is similar to Eq. (46) for $C_{1}(z)$, except that $C_{3}(z)=-\left(40 \epsilon_{3} \eta_{1} \eta_{2}^{2}\right) /(3|\beta| \beta)$ for $z \geq z_{c}$.

[67] Note that the collision-induced position shift experienced by soliton 2 due to the effects of Kerr nonlinearity is [14, 16, 17]: $\Delta y_{22}^{(0)}=-4 \eta_{1} /(\beta|\beta|)$. Therefore, Eq. 52 for $C_{4}(z)$ is similar to Eq. (48) for $C_{2}(z)$, except that $C_{4}(z)=-4 \eta_{1} /|\beta|$ for $z_{c}-1 /(2|\beta|) \leq z \leq z_{c}+1 /(2|\beta|)$. 